\title{
Evolutionary Algorithm for Multiobjective Optimization Based on Density Estimation Ranking
}

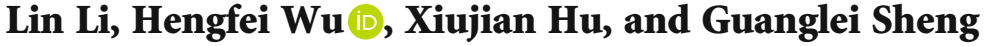 \\ Department of Electronics and Information Engineering, Bozhou University, Bozhou Anhui, China \\ Correspondence should be addressed to Hengfei Wu; whf520315000@163.com
}

Received 9 May 2021; Revised 1 June 2021; Accepted 19 June 2021; Published 6 July 2021

Academic Editor: Shan Zhong

Copyright ( 2021 Lin Li et al. This is an open access article distributed under the Creative Commons Attribution License, which permits unrestricted use, distribution, and reproduction in any medium, provided the original work is properly cited.

\begin{abstract}
In the past few decades, a number of multiobjective evolutionary algorithms (MOEAs) have been proposed in the continue study. As pointed out in some recent studies, the performance of the most existing MOEAs is not promising when solving different shapes of Pareto fronts. To address this issue, this paper proposes an MOEA based on density estimation ranking. The algorithm includes density estimation ranking to shift the reference solution position, calculating the density of candidate solutions and ranking by the estimated density value, to modify the Pareto dominance relation and for handling complicated Pareto front. The result of this ranking can be used as the second selection criterion for environmental selection, and the optimal candidate individual with distribution and diversity information is selected. Experimental results show that the proposed algorithm can solve various types of Pareto fronts, outperformance several state-of-the-art evolutionary algorithms in multiobjective optimization.
\end{abstract}

\section{Introduction}

Multiobjective optimization problems (MOPs) are common in the real-life, e.g., robotics [1], urban bus transit route network design problem [2], smart grids [3], and electricity selling market [4]. These problems aim to optimize more than two conflicting objectives at the same time, which can be mathematically formulated clearly depict as follows.

$$
\begin{array}{cc}
\text { Min } & F(x)=\left(f_{1}(x), f_{2}(x), \cdots, f_{m}(x)\right) \\
\text { s.t } & x \in X
\end{array}
$$

where $\mathbf{x}$ denotes the decision vector, $X \subseteq R^{n}$ is called the decision space, and $F(x) \in R^{m}$ is the objective vector of $\mathbf{x}$, which consists of $m$ objective functions of $f_{i}(x), i=1,2, \cdots, m$.

However, owing to the conflicting nature of multiple objectives, there is not an algorithm that obtains a single optimal solution that can optimize all objectives. Instead, some solutions can be obtained as a trade-off between different objectives, called the Pareto set (PS). The PS is termed the
Pareto front $(\mathrm{PF})$ in the objective space. To approximate the Pareto optimal set, greatly quantities of multiobjective evolutionary algorithms (MOEAs) have been proposed to solve MOPs in the past several decades. These algorithms can be roughly classified into three categories.

The first category is the dominance-based MOEAs, which keep the nondominated solution and remove the dominated solution in the population [5]. There are mainly two types of dominance-based approaches: the first type of Pareto dominance-based MOEAs, which Pareto dominance-based mechanisms are adopted to distinguish and select candidate solutions. For example, the elitist nondominated sorting genetic algorithm (NSGA-II) [6], the Pareto envelop-based selection algorithm II (PESAII) [7], and the strength Pareto evolutionary algorithm 2 (SPEA2) [8] are representative MOEAs of this type, where all nondominated solutions are firstly identified, and then the other strategy is used to make selections among the nondominated solutions to maintain the population diversity.

The second type of dominance-based approaches, where it improves the selection pressure of the environment by 


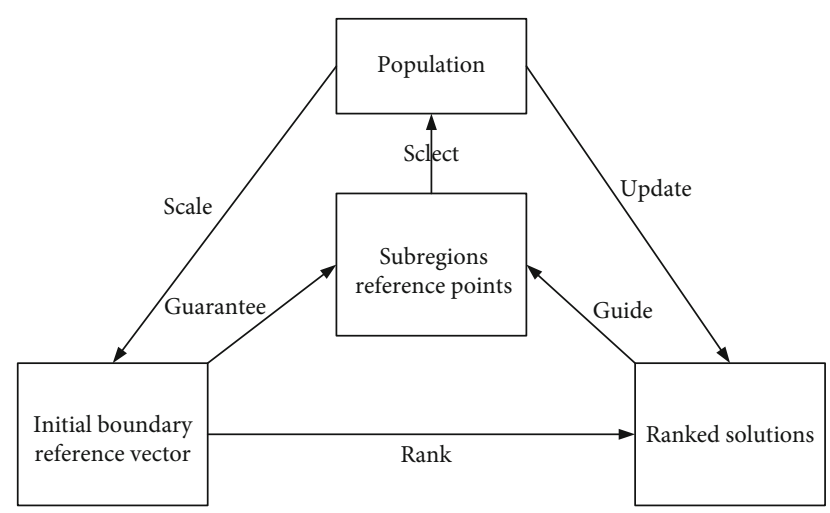

FIGURE 1: The relationships between the four sets in DEREA, i.e., the population $P$, the initial boundary reference vectors $R$, the ranked solutions $S$, and the subregions boundary reference point set $R^{\prime}$. First, the initial vectors of scale in $R$ according to the dimension of the population $\mathrm{P}$ and the extreme solutions is selected by the boundary reference vector and obtain the rank value 0 . Then, the ranked solutions $S$ close the true $\mathrm{PF}$ as subregions boundary reference point $R^{\prime}$. Finally, the DER selects subregions optimal solution and rank.

changing the Pareto dominance, thus does not the need for other selection strategies. Remarkable MOEAs based on change Pareto dominance have included $\varepsilon$-MOEA [9], MO_Ring_PSO_SCD) [10], and HTL-PSO [11].

The second category is commonly known as the decomposition-based MOEAs, which decomposes a complex MOP into a number of subproblems and optimizes them simultaneously [12]. There are mainly divided MOEAs into two types of decomposition-based approaches. In the first type of decomposition-based approaches, an MOP is divided into a group of single-objective optimization problems (SOPs) [13]. The MOEA/D proposed in [14] is a simple and generic MOEA based on decomposition, which introduces a set of weight vectors to manage and select candidate solutions. [15] proposed a reference vector-guided evolutionary algorithm, which MOPs are divided into a set of SOPs by the reference vector; in this way, the candidate solutions will effectively converge to the optimal solutions of each SOPs without considering the conflict between different SOPs.

In the second type of decomposition-based approaches, an MOP is divided into a set of sub-MOPs or subregions. For instance [16], the during decades proposed include reference point-based nondominated sorting genetic algorithm III (NSGA-III) [17], inverse modeling-based MOEA (IMMOEA) [18], and the $k$-means clustering method divides the population into multiple subpopulations in GSMPSO-MM [19], and learning to-decompose paradigm adaptively sets the decomposition method with the Pareto front $[20,21]$ that proposed a MOEAD-M2M, which divided MOP into a group of simple subproblems and solving these in a collaborative manner, population diversity will be achieved by this way. The proposed algorithm termed as SPEA/R, which divides objective space into a set of subregions, and individuals in every subregion are driven toward target direction [22].

The third category is known as the indicator-based approaches, where performance indicators of solution qual-

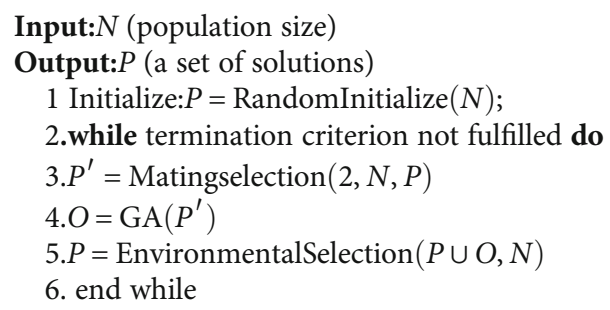

Algorithm 1: Framework of DEREA.

ity measurement are adopted as selection criteria in the environmental selection. Representative MOEAs of this type contain $S$ metric selection evolutionary multiobjective optimization algorithm (SMS-EMOA) [23], generational distance and $\varepsilon$-dominance based MOEA (GDE-MOEA) [24], indicator-based evolutionary algorithm [25], a dynamic neighborhood MOEA based on hypervolume indicator (DNMOEA/HI) [26], an inverted generational distance (IGD) indicator-based evolutionary algorithm [27], and R2 indicator-based many-objective metaheuristic-II (MOMBIII) [28], where the environment selection strategies are designed based on a predefined the hypervolume (HV) indicator, the generation distance (GD), binary indicator, and the R2 indicator, respectively. The hype suggested an HV indicator-based MOEA [29], where it uses Monte Carlo simulation to approximate the exact HV values. The AR-MOEA is based on the enhanced inverted GD (IGD-NS) indicator [30], where a reference point adaptation method is to adjust a set of reference points based on the indicator calculation of candidate solutions.

There are a few other algorithms that are not included in the above three main categories. For example, a decision variable analysis MOEA (MOEA/DVA) has been proposed based on decomposition and differential evolution (DE) [31]. The MOEA/D-CMA suggested in [32] in which it uses $\mathrm{DE}$ and covariance matrix in the MOEA based on decomposition, and DREA has been proposed in the [33], which is based on diversity ranking. More recently, a new algorithm has been suggested based on fuzzy and decomposition for multiobjective optimization (MOEA-MCD) [34]. An enhanced two-archive algorithm (Two_Arch2) has been suggested based on different selection principles (indicator and dominance) in [35], which design a new Lp-norm maintenance for diversity and convergence. A one-by-one selection strategy has been provided in [36], where main approach is in the environment selection strategy to selection offspring individuals one by one, which is based on a computationally efficient convergence indicator to increase the selection pressure towards the Pareto optimal front.

These MOEAs are suggested to solve MOPs in the literature, and the experimental results verify that many MOEAs can well balance the convergence and diversity of population. And yet, it have been pointed out in some recent studies that the most existing MOEAs for the optimization problems show performance that can strongly depend on PF shape [37, 38]. In other words, some MOEAs are merely solving a type of PFs of MOP and show versatility that is not good when these MOEA 


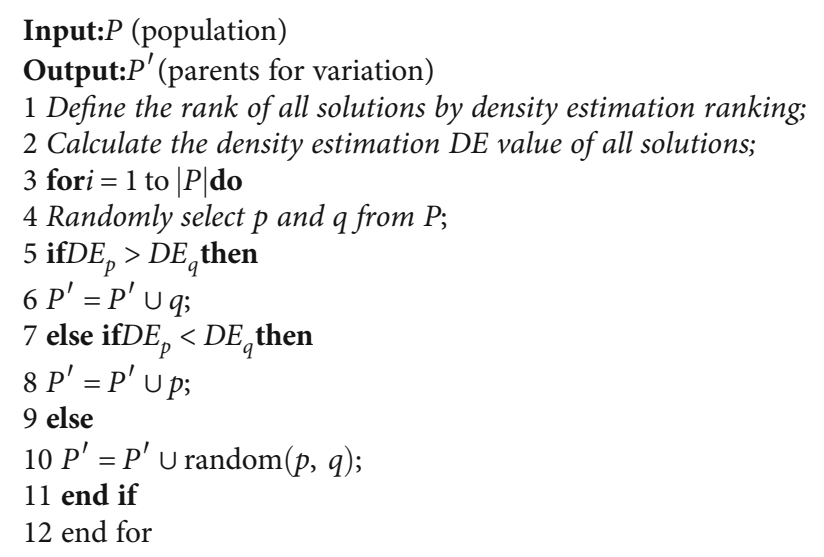

Algorithm 2: Mating selection.

optimizing other types PFs of MOP. This is due to the most MOEAs that are very sensitive to PF shapes.

For the above issues, this paper has been suggested a density estimation ranking- (DER-) based evolutionary algorithm for multiobjective optimization, called DEREA. The main contributions of this work included as follows.

(1) The current work has much of the study primary selection criterion (i.e., modifying the Pareto dominance approach) in Pareto-based algorithms to solved multiobjective, and this paper enhances the Pareto-based algorithm performance by the other selection criterion (modifying the diversity maintenance mechanism). This paper has been suggested a DER approach in order to enhance Pareto-based algorithm suitable for multiobjective optimization. The DER simultaneously includes the convergence and distribution information of individuals to enhance MOEA performance

(2) The basic approach of DER is simple, considering the contribution of individuals to population convergence in sparse area, and the DER shifts poor convergence individuals to density crowded area. In this way, these individuals with poor convergence performance will be given a high density value, which is easier to be eliminated in the evolution process, so improving the efficiency of the algorithm. In addition, the calculated density value is used directly for ranking, and the calculation cost is negligible

The rest of this paper is organized as follows. In Section 2, the details of the proposed algorithm DEREA are described. The empirical results of DEREA compared with several classical MOEAs are presented in Section 3. Finally, conclusion and future work are given in Section 4.

\section{The Proposed DEREA Preparation}

2.1. The Framework of DEREA. The proposed DEREA has similar framework as NASA-II, except that DER is adopted as the second select criterion and the boundary reference vectors to manage diversity and convergence for various types of the Pareto fronts. In general, there are four main solution sets maintained in DEREA, i.e., the population $P$, the initial boundary reference vectors $R$, the ranked solutions $S$, and the subregions boundary reference point set $R^{\prime}$. To be specific, the population $P$ contains the candidate solutions, guaranteeing uniform distribution of the candidate solutions in $P$ by the initial boundary reference vector set $R$ and the ranked solutions $S$ as final output reflects the PF and guides the boundary reference points adaptation come into being, and the subregions boundary reference point set $R^{\prime}$ is used in the DER-based selection in the population $P$, where the relationships between the four solutions are described in Figure 1.

As presented in Algorithm 1, the main framework of DEREA consists of the following steps. Firstly, an initial population $P$ of size $N$ is randomly generated. Then, in the main loop, the mating selection is carried out to construct the $P^{\prime}$ (mating pool) based on the ranking results, where mating selection will be detail described in Algorithm 2. The $O$ (offspring population) has been generated by genetic algorithm operations. The parents and their offspring solutions are obtained by the environment selection. Finally, the best $N$ solutions are selected by the environment selection, and the new population comes into being.

2.2. Offspring Creation. In the suggested DEREA, the widely used genetic operators [39], i.e., the polynomial mutation [40] and the SBX (simulated binary crossover) [41], are introduced to combine with the binary tournament selection [42], which constructs the mating selection method to generate the offspring population. Thus, initial population $P$ includes $N$ individuals, and a number of $N / 2$ couple of parents are randomly generated, i.e., each of the $N$ individuals has same probability to participate in the reproduction procedure. Algorithm 2 details the binary tournament selection and density estimation in the main loop. This is possible partly thanks to boundary reference vector strategy (it will be explained in detail later), which can capture the boundary 


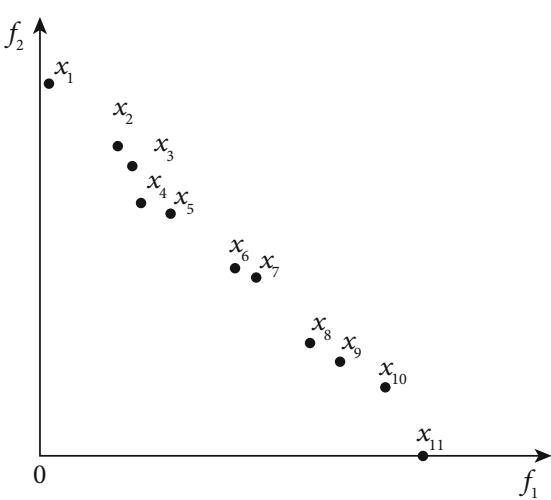

(a) Candidate population

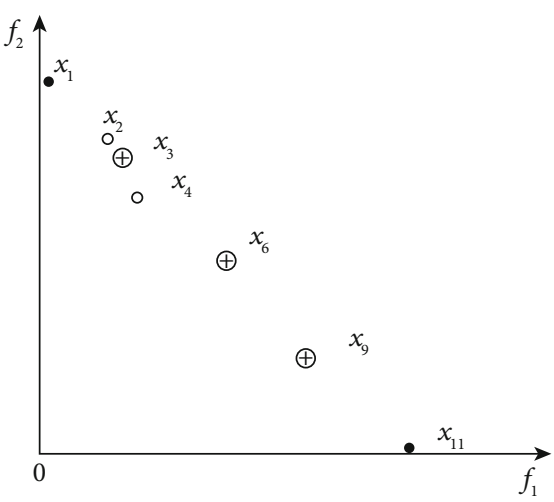

(b) Selected solutions

Figure 2: An illustrative example to show the advantage of DER over SDE.

of the PF to manage the diversity effectively ( $m$ boundary reference vector search $m$ solutions).

2.3. Density Estimating. In a population, an individual density represents the degree of crowding in the region where the individual is located. Density estimation plays a fundamental role in the select Pareto solution set. Ther are various density estimation strategies in the MOEAs. For instance, the SPEA2 uses a nearest neighbor density estimation strategy to calculation the density of an individual [8]. NSGA-II gives an expression in the density of an individual by calculating crowing distance [6]. Most grid-based MOEAs, for example, the dynamic multiobjective evolutionary algorithm [43] and PESAII, calculat the number of individuals in the hyperbox to reflect the density of an individual; yet, some scholars proposed to use a set of hyperboxs to describe the density of an individual based on the degree of individual crowding $[44,45]$.

Despite the various strategies of density estimation, this all measures the similarity of degree between individuals in a population. Generally, lower density individuals are preferable when two individuals are nondominant individuals in the population. DE contributes greatly to the Pareto solution set in the MOEA-based Pareto dominates. Therefore, this paper introduces enhance shift-based density estimating (SDE) [46] to maintain the diversity and convergence of population.

Simply speaking, if there is an individual performing better than individual $p$ for an objective, this objective will be moved to the same position of $p$ in the population; otherwise, its position will remain unchanged. Formally, the density $D$ $(p, P)$ of a candidate solution $p$ in the population $P$ can be expressed as follows:

$$
D(p, P)=D\left(\operatorname{dist}\left(p, q_{1}^{\prime}\right)+\operatorname{dist}\left(p, q_{2}^{\prime}\right)+\cdots+\operatorname{dist}\left(p, q_{N-1}^{\prime}\right)\right),
$$

where $\operatorname{dist}\left(p, q_{i}^{\prime}\right)$ denotes the calculation of the Euclidean distance between individual $p$ and selected solution $q_{i}^{\prime}, N$ denotes the size of population $P$, and $q_{i}^{\prime}$ is the shift version of an indi-

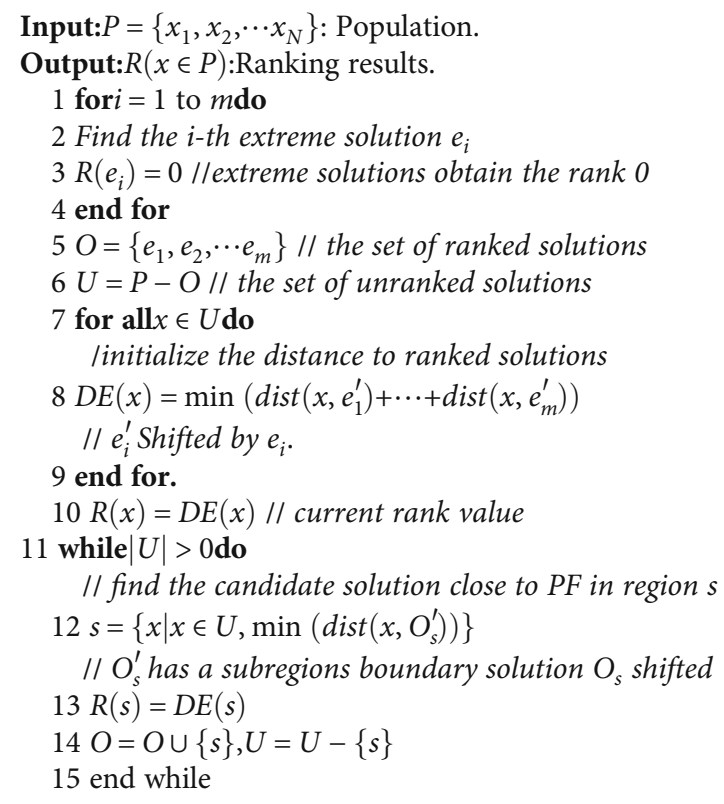

Algorithm 3: Density estimation ranking.

vidual $q_{i}\left(q_{i} \in P\right.$ and $\left.q_{i} \neq p\right)$, which is defined as follows:

$$
q_{i(j)}^{\prime}=\left\{\begin{array}{ll}
q_{i(j)} & \text { otherwise } \\
p_{(j)} & \text { if } q_{i(j)}<p_{i(j)}
\end{array}, j \in(1,2,3, \cdots, m)\right.
$$

where $p_{(j)}, q_{i(j)}$, and $q_{i(j)}{ }^{\prime}$ denote the $j$ th objective value of individual $p, q_{i}$, and $q_{i}^{\prime}$, respectively, and $m$ denotes the number of objectives.

The following sections will describe the two main components of suggested DEREA, i.e., the density estimation ranking and environment selection.

2.4. Ranking the Solutions. When a great number of objectives are involved, the proportion of nondominant individuals in the population becomes quite large. In extreme cases, all individuals in the population may become 


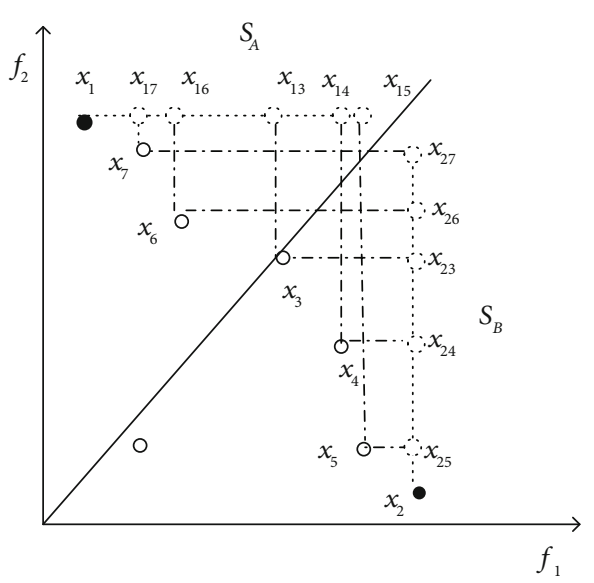

(a)

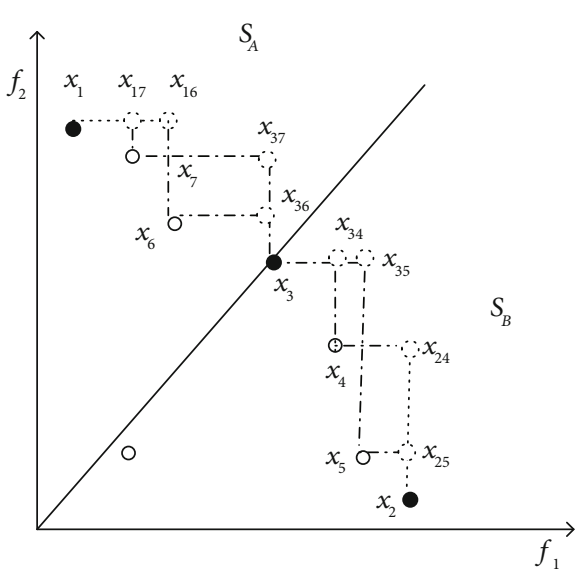

(b)

FIGURE 3: Illustration of the first two iterations of the ranking approach.

nondominated with each other. In this case, the individual density will play a dominant or even unique role in the selection process of the algorithm to distinguish them. It can be clearly known that only the individual with both good diversity and convergence has a low crowding value, when using the density estimation method calculation of an individual density. Certainly, either individual with poor diversity or poor convergence has some neighbors. Individual has a high crowding when an individual has with both poor diversity and poor convergence. Therefore, we want to select the individual with the smallest density value by ranking.

Based on the density estimation strategy, the DER is proposed in this subsection. Supposing a population $P$ contains $N$ solutions, the task of DER is to distribute a rank value to each candidate solution in $P$, using the calculation density value of the individual as the rank value and describes its quality in terms of both diversity and convergence. In this study, hoping to get a smaller rank value, the best solutions accept the rank 0 . Therefore, when applying density estimation to evolutionary algorithms, candidate solutions with smaller rank values are more apt to survive in the mating selection and the environmental selection.

Compared to the SDE, DER adopts more comprehensive information to select a solution set. Consider an example as shown in Figure 2, where $x_{1}-x_{11}$ is a candidate population. In the case that five out of the eleven candidate solutions are to be selected for next generation, SDE to select solutions is $\left\{x_{1}, x_{2}, x_{3}, x_{4}, x_{11}\right\}$. The DER chooses evenly distributed solutions that are $\left\{x_{1}, x_{3}, x_{6}, x_{9}, x_{11}\right\}$, which are the best candidate solutions in terms of diversity and convergence. The reason for this phenomenon is that SDE selected the enough solutions in the one region. However, during the selection process by DER category, solutions divide a complex PF into a number of subregions, then selecting the optimal solution in every subregion.

DER works like a selection operator which selects unranked solutions iteratively. In its main loop, there are two sets $O$ and $U$, which are composed of ranked solutions and unranked individuals, respectively. Initially, $O$ is the set

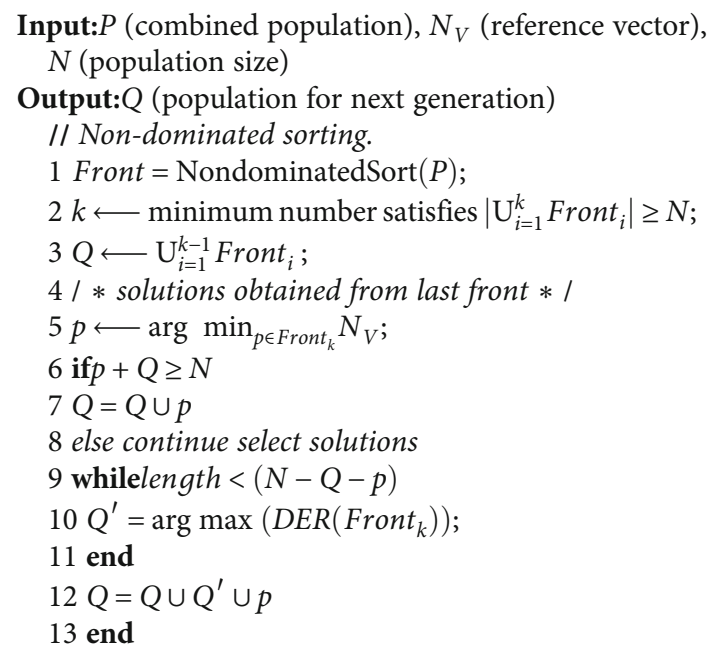

Algorithm 4: Environmental selection.

of extreme solutions $e_{1}, e_{2}, \cdots, e_{m}$, and the extreme solutions are selected by the boundary reference vector. Capturing the boundary of the PF can be beneficial for the next step of the selection optimal solutions. This part is not the main contribution of this paper, and thus the method proposed in [16] is employed here, and the extreme solutions in $O$. These are the most boundary solutions of PF, facilitating the DER method to rank the population by PF boundary. However, $U$ includes all remaining solutions:

$$
O=\left\{e_{1}, e_{2}, \cdots, e_{m}\right\}, U=P-O \text {. }
$$

After the initialization, we select a solution $s$ with the smallest individual density value from $U$ according to the density estimation method. The $s$ is selected processing 
which is defined as follows:

$$
s=\arg \min _{x \in U} D\left(x, e_{O}\right),
$$

where $e_{O}$ denotes the extreme solutions by boundary reference vectors, and $D\left(x, e_{O}\right)$ denotes calculate density of an individual $x$ in the population. This step is designed to maintain the diversity. Since $s$ is close to PF, selecting the solutions has high quality in terms of both convergence and diversity. The estimated individual density value as the ranking value is moved to $O$. Then, the $s$ is divided in the entire region into two subregions, and selecting optimal solution $s$ in every subregions is moved to $O$. These operations are repeated until $U$ is empty.

All the details of DER have been discussed in above. Algorithm 3 provides the pseudocode of DER. The result of ranking $x$ is denoted by $R(x)$. Lines 1 to 4 use the boundary vector to select the extreme solutions and obtain the rank 0 . In lines 5 to $9, O$ and $U$ are initializing, and the minimum density value to the rank solutions after the shifted position of each solution in $U$ is initializing. Then, loop in lines 11 to 16 iteratively ranks the remaining solutions. Specifically, in constant iteration, the promise solution $s$ is first found in line 12 . The density value of the promise solution is its rank value in line 13. Finally, in line 14, we move $s$ from $U$ to $O$. Since the key individual is inserted every time the loop ranks, the density of unranked individuals will gradually decrease; so, previously ranked solutions always get higher rank value than those ranked in the subsequent iterations.

To clearly understanding the ranking of process, Figure 3 depicts an illustration of its first two iterations. The circle in the figure represents the solutions in the entire population, where filled circle (i.e., $x_{1}$ and $x_{2}$ ) represents the extreme solutions selected by the boundary vector, and the dotted circle indicates the position after the extreme solution is shifted when calculating the individual density. $x_{13}, x_{14}, x_{15}, x_{16}, x_{17}$, $x_{23}, x_{24}, x_{25}, x_{26}$, and $x_{27}$ denote the position after shifting when calculating the individual density, where $x_{13}$ and $x_{23}$ represent the position after $x_{1}$ and $x_{2}$ shift when calculating the density of individual $x_{3}$. Firstly, calculating the density of unranked individual $p$ using the extreme solutions selected by the boundary vector, where extreme solution position is shifted by the density estimation approach and then selected the individual with the smallest density value as the promise solution $s$. Then, the individual $x_{3}$ with the smallest density value is selected as $s$ by comparing the density values of all individuals. Meanwhile, it gets rank value that is the density value. Secondly, bipartition the objective space into two subregions $S_{A}$ and $S_{B}$ by $x_{3}$. Then, $x_{1}$ and $x_{3}$ are the boundary extreme solutions of subregion $S_{A}$, and $x_{2}$ and $x_{3}$ are the boundary extreme solutions of subregion $S_{B}$; in the second iteration to select key individual in each subregion, the key individual with the smallest density value is the promising solution, and it gets the rank and is moved to $O$. After these iterations, obtaining the rank solutions is $x_{1}, x_{2}, x_{3}, x_{4}$, and $x_{7}$.

In the above example, it is easy to understand that the selection pressure is usually greater in the previous stage, but the selection pressure gradually decreases with more and more
TABLE 1: Pareto fronts of the test instances.

\begin{tabular}{lcc}
\hline Problem & \multicolumn{2}{c}{ Pareto front } \\
\hline DTLZ1 & Linear & Regular \\
DTLZ2-4 & Concave & \\
WFG4-9 & Concave & \\
DTLZ5-6 & Mostly degenerate & \\
DTLZ7 & Disconnected & Irregular \\
IDTLZ1-2 & Inverted & \\
WFG1 & Sharp tails & \\
WFG2 & Disconnected & \\
WFG3 & Mostly degenerate & \\
\hline
\end{tabular}

TABLE 2: Setting of the population size.

\begin{tabular}{lcc}
\hline$M$ & Division & Population size \\
\hline 2 & $(15,0)$ & 100 \\
3 & $(13,0)$ & 105 \\
\hline
\end{tabular}

solutions that are selected and ranked. Thus, previously ranked solutions have a high quality, because they have survived in the fierce selection competition. And they will be assigned a larger rank values compared to those ranked in the later stage.

2.5. Environment Selection. The DEREA is similar to the most existing MOEAs, which adopt elite strategies to make environmental selections for the combined population of each generation's parent and offspring candidate solutions.

The procedure of the DER-based environmental selection is given in Algorithm 4. The main loops are as follows: Firstly, before the DER to selecting the candidate solution, making the combined population is first sorted by adopting the high efficiency nondominated rank. Then, population in all candidate solutions from nondominated fronts in the first $k-1$ was selected directly for the next generation (line 3 in Algorithm 4). Secondly, the corresponding solution is selected by the boundary vector to enter the next generation (line 5 in Algorithm 4). Finally, because $p+Q<N$, continuing to select $N-(p+Q)$ candidate solutions from the population by DER (line 11and 13 in Algorithm 3). The DER is used to select candidate solutions in the $k$ th front Front ${ }_{k}$, where $k$ defined the minimum number it satisfies $\mid \mathrm{U}_{i=1}^{k}$ Front $_{i} \mid \geq N$. Each candidate solutions in the Front ${ }_{k}$ and its contribution to Front ${ }_{k}$ on DER are calculating the Euclidean distance. Then, we will delete the solution with small contribution for efficiency, recalculate the contribution of the remaining solution in the Front $_{k}$, and repeating this process until the number of remaining solutions in $U_{i=1}^{k}$ Front $_{i}$ reaches $N$.

It is noteworthy that although the selection process in the most Pareto-based dominance MOEAs are guided by the density estimation value, the motivation of employing DER in DEREA is very different. In DEREA, we transfer position of each individual by SDE mechanism, then rank of each individual by density estimation value. The most existing 


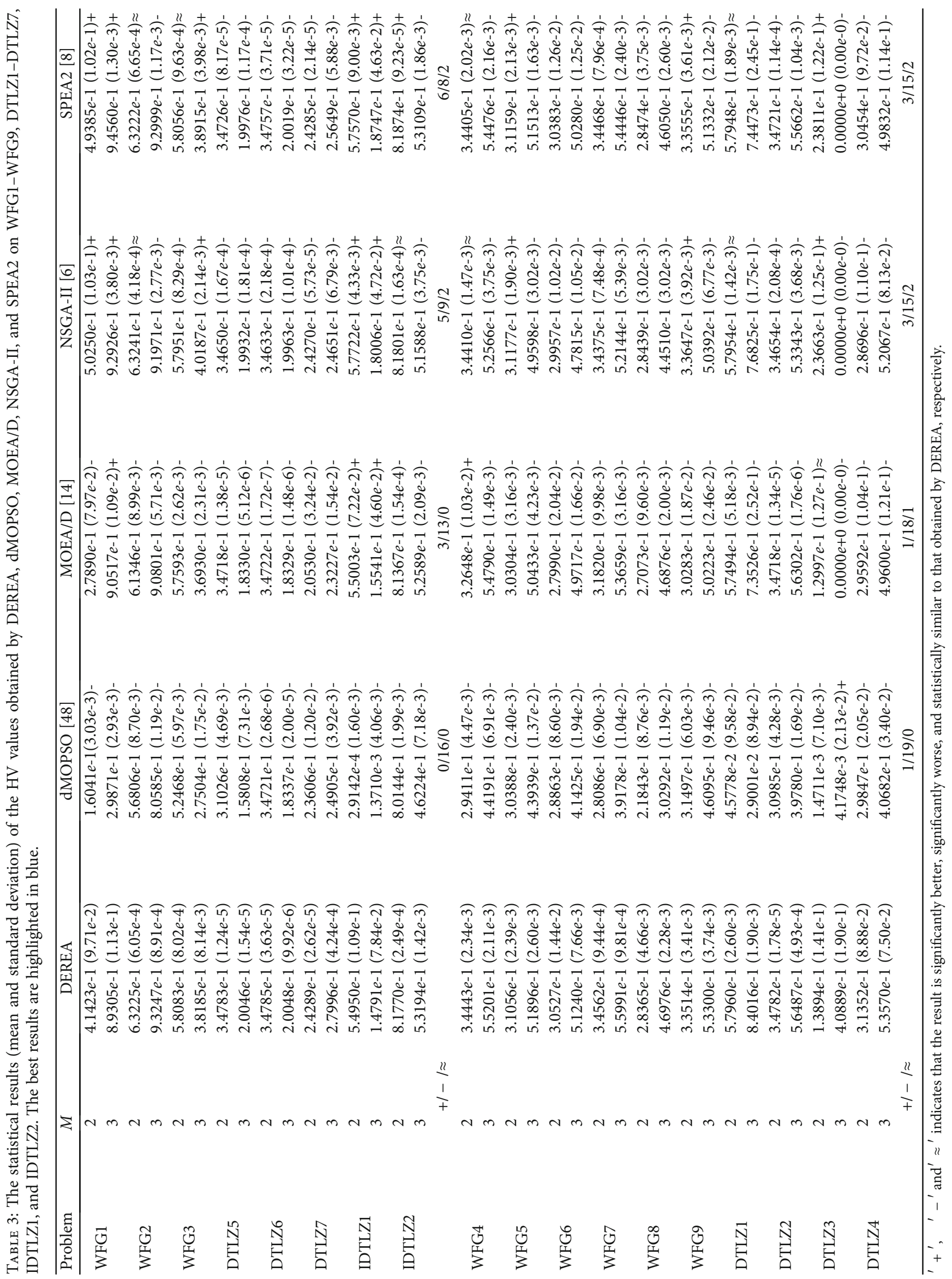




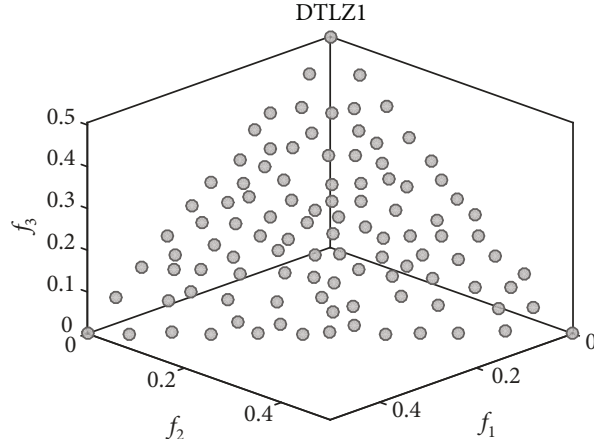

(a) DEAER

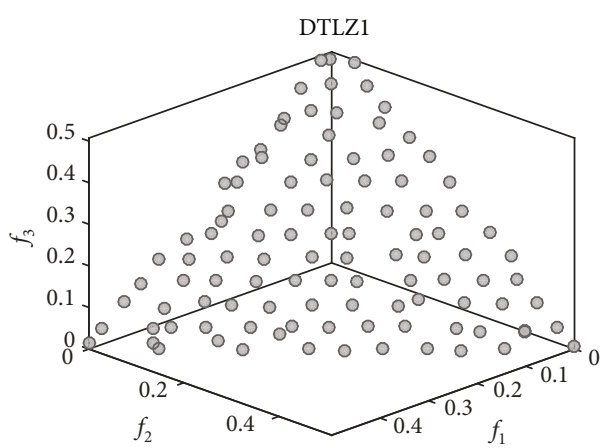

(c) MOEA/D [14]

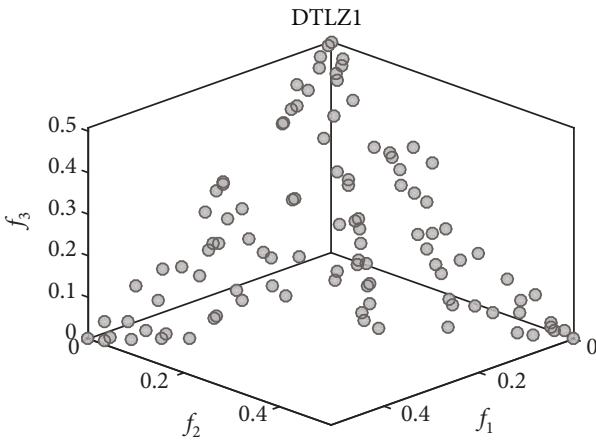

(e) NSGA-II [6]

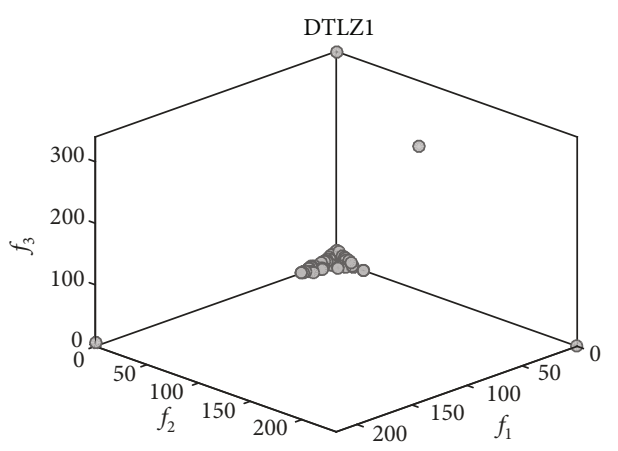

(g) SMEA [51]

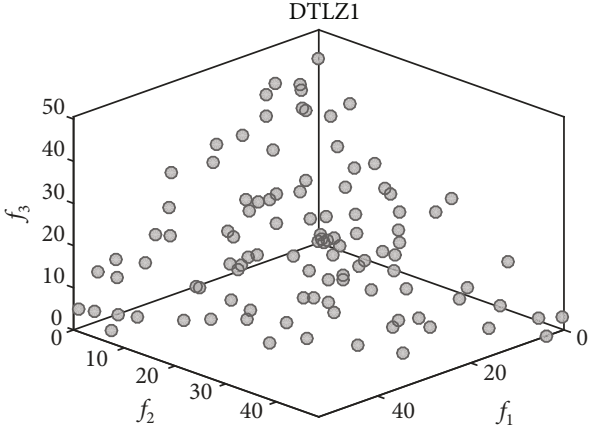

(b) dMOPSO [48]

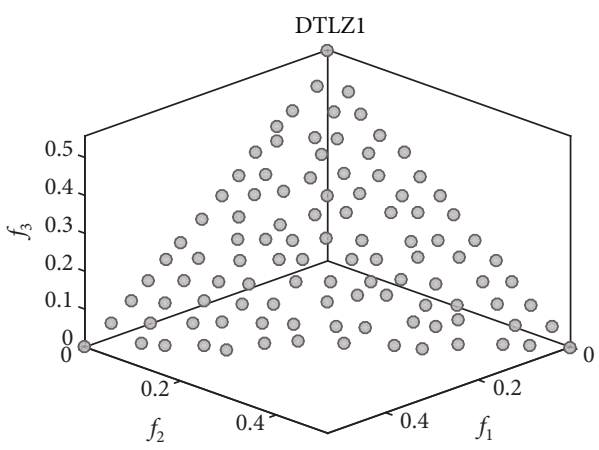

(d) MOEA/DDE [50]

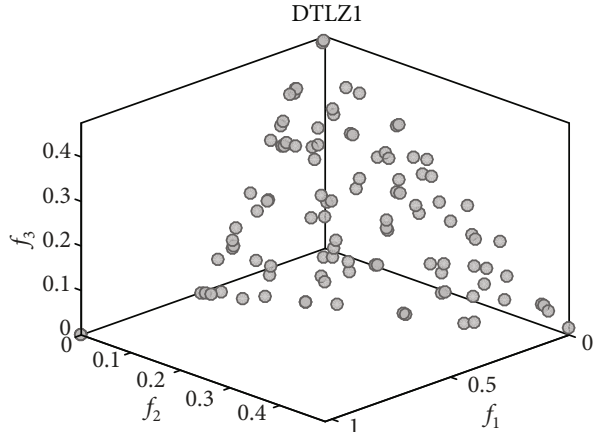

(f) PESAII [7]

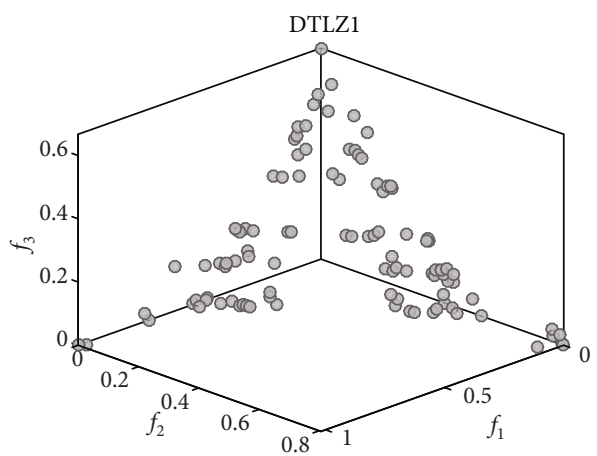

(h) SPEA2 [8]

FIGURE 4: The nondominated solutions obtained by DEREA, dMOPSO [48], MOEA/D [14], MOEA/DDE [50], NSGA-II [6], PESAII [7], SMEA [51], and SPEA2 [8] on DTLZ1 problem with 3-objective. 


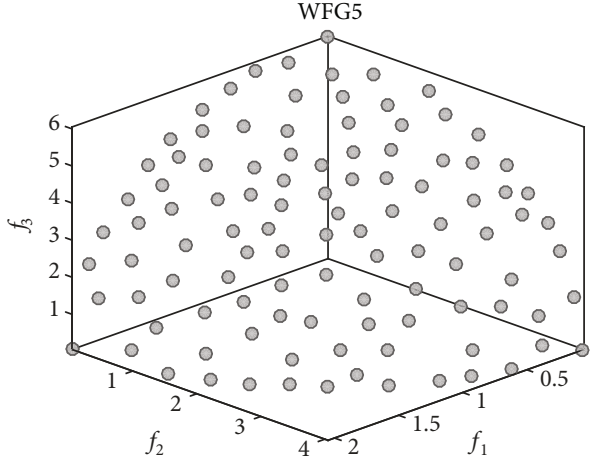

(a) DEAER

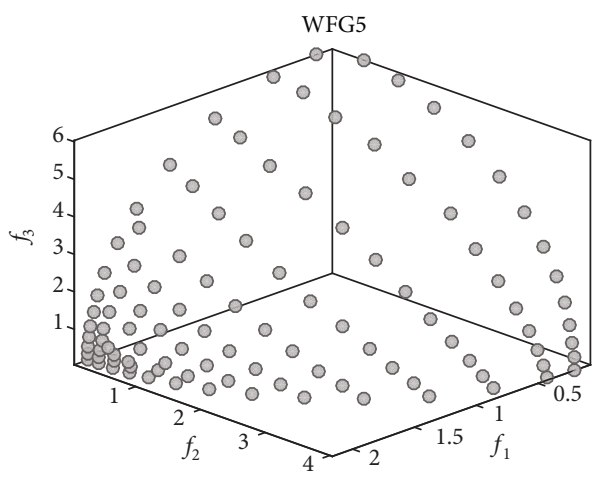

(c) MOEA/D [14]

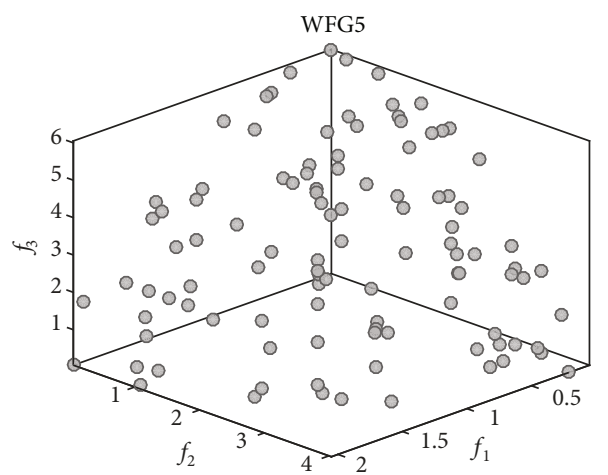

(e) NSGA-II [6]

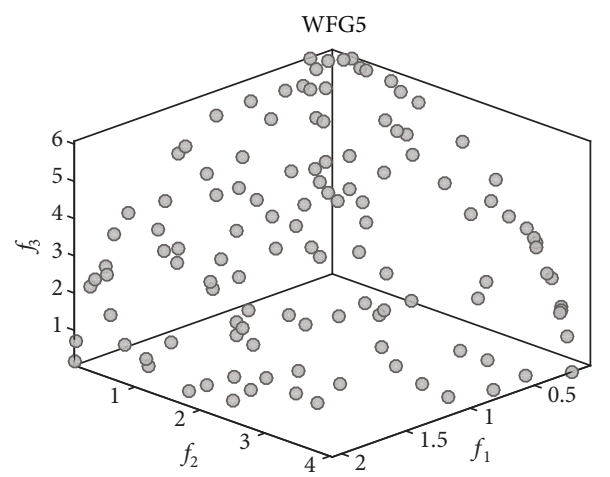

(g) SMEA [51]

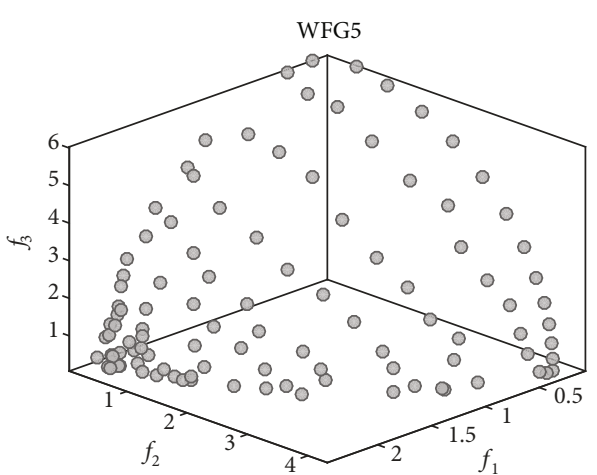

(b) dMOPSO [48]

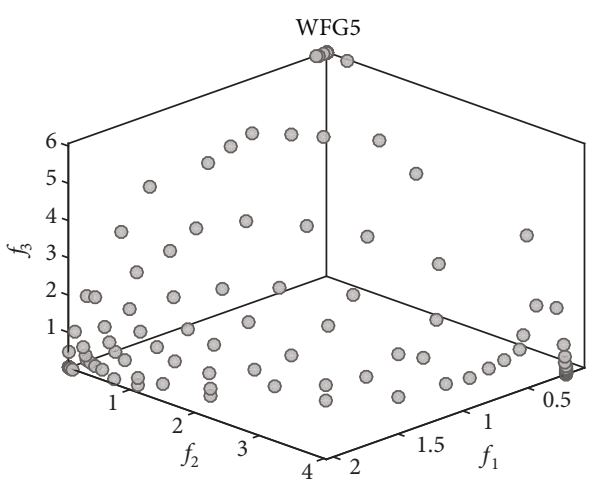

(d) MOEA/DDE [50]

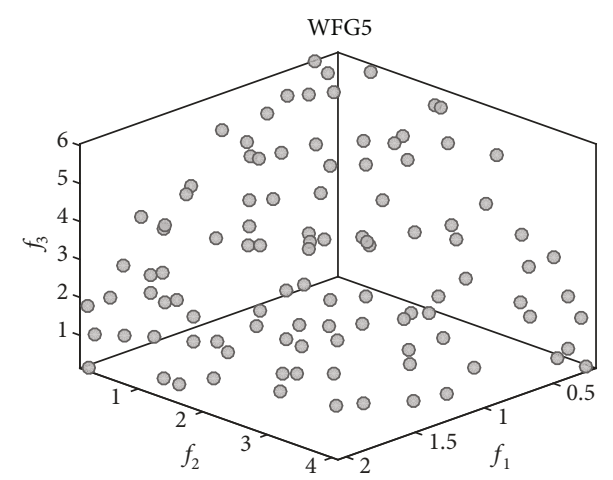

(f) PESAII [7]

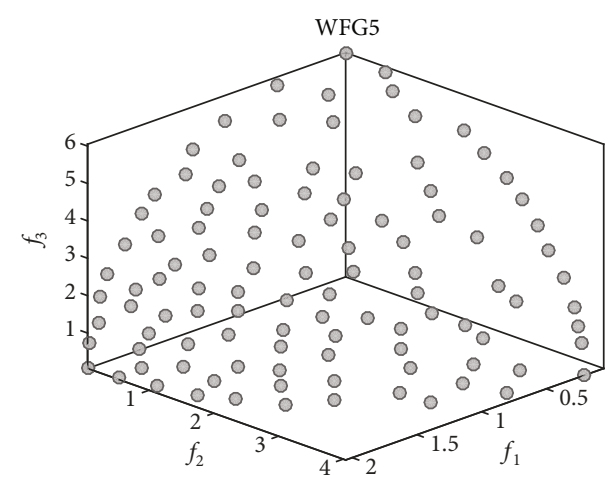

(h) SPEA2 [8]

FIgURE 5: The nondominated solutions obtained by DEREA, dMOPSO [48], MOEA/D [14], MOEA/DDE [50], NSGA-II [6], PESAII [7], SMEA [51], and SPEA2 [8] on WFG5 problem with 3-objective. 


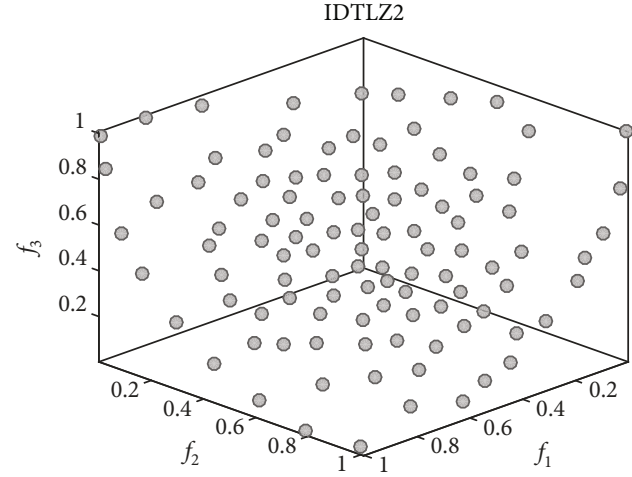

(a) DEAER

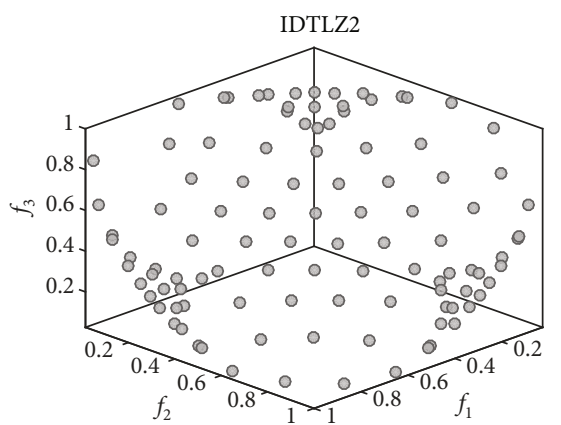

(c) MOEA/D [14]

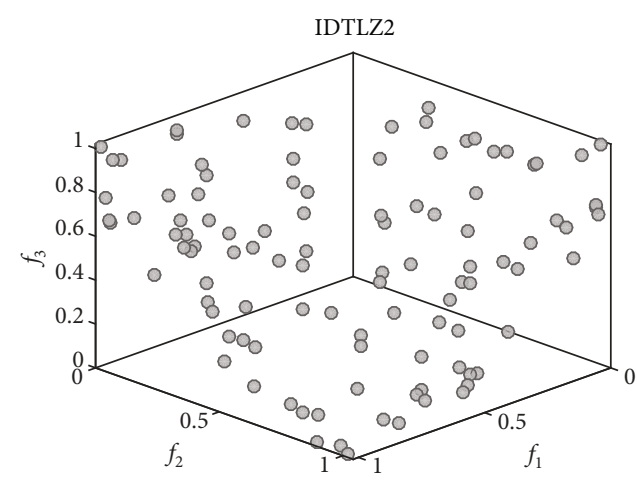

(e) NSGA-II [6]

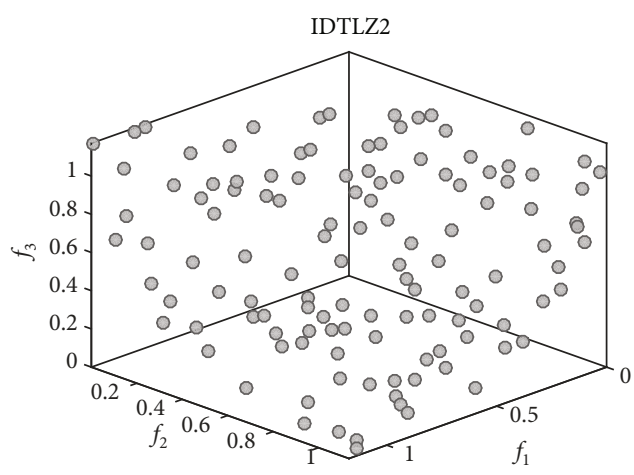

(g) SMEA [51]

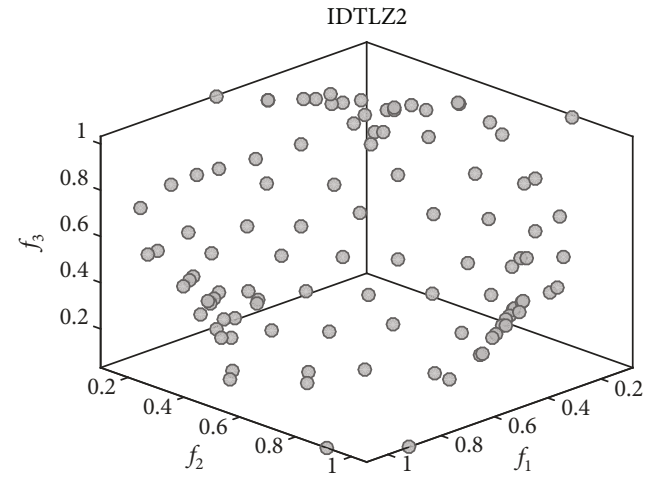

(b) dMOPSO [48]

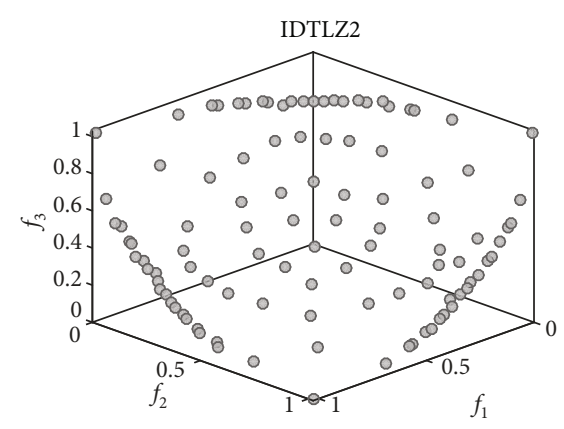

(d) MOEA/DDE [50]

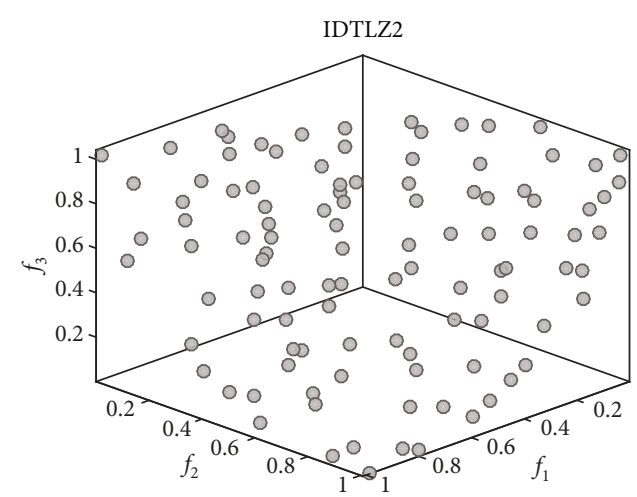

(f) PESAII [7]

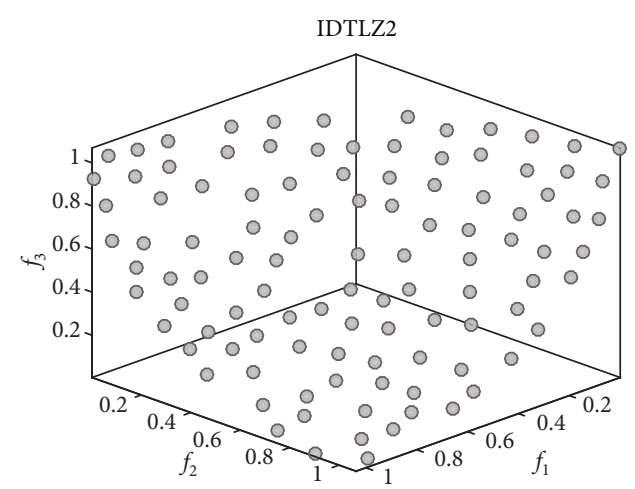

(h) SPEA2 [8]

Figure 6: The nondominated solutions obtained by DEREA, dMOPSO [48], MOEA/D [14], MOEA/DDE [50], NSGA-II [6], PESAII [7], SMEA [51], and SPEA2 [8] on IDTLZ2 problem with 3-objective. 
TABLE 4: The statistical results (mean and standard deviation) of the IGD values obtained by DEREA, PESAII, dMOPSO, NSGA-II, and MOEA/DDE on UF1-UF10. The best results are highlighted in blue.

\begin{tabular}{lcccccc}
\hline Problem & $M$ & DEREA & PESAII [7] & dMOPSO [48] & NSGA-II [6] & MOEA/DDE [50] \\
\hline UF1 & 2 & $7.6648 e-2(1.86 e-2)$ & $1.2949 e-1(4.29 e-2)-$ & $2.1650 e-1(6.57 e-2)-$ & $8.2028 e-2(1.29 e-2) \approx$ & $3.8810 e-2(1.64 e-2)+$ \\
UF2 & 2 & $2.4740 e-2(4.42 e-3)$ & $3.7279 e-2(1.57 e-2)-$ & $5.3844 e-2(6.62 e-3)-$ & $2.7868 e-2(6.93 e-3) \approx$ & $2.1435 e-2(8.96 e-3)+$ \\
UF3 & 2 & $2.1154 e-1(4.33 e-2)$ & $2.5232 e-1(4.31 e-2)-$ & $3.3960 e-1(2.08 e-2)-$ & $2.3749 e-1(4.41 e-2)-$ & $2.1562 e-1(4.37 e-2) \approx$ \\
UF4 & 2 & $4.0734 e-2(1.72 e-3)$ & $4.6986 e-2(1.70 e-3)-$ & $9.7788 e-2(7.03 e-3)-$ & $4.2393 e-2(1.25 e-3)-$ & $5.9141 e-2(5.89 e-3)-$ \\
UF5 & 2 & $2.7954 e-1(6.80 e-2)$ & $3.9438 e-1(1.06 e-1)-$ & $1.1205 e+0(2.25 e-1)-$ & $3.2780 e-1(8.90 e-2)-$ & $4.5750 e-1(1.02 e-1)-$ \\
UF6 & 2 & $2.0051 e-1(7.66 e-2)$ & $3.1073 e-1(1.24 e-1)-$ & $9.4042 e-1(7.82 e-2)-$ & $2.0025 e-1(8.76 e-2) \approx$ & $3.1438 e-1(1.52 e-1)-$ \\
UF7 & 2 & $8.6449 e-2(1.17 e-1)$ & $2.5804 e-1(1.88 e-1)-$ & $1.1975 e-1(2.68 e-2)-$ & $1.0507 e-1(1.34 e-1) \approx$ & $2.2722 e-2(4.22 e-2)+$ \\
UF8 & 3 & $1.4673 e-1(4.44 e-2)$ & $2.2468 e-1(5.76 e-2)-$ & $2.9173 e-1(3.77 e-2)-$ & $2.4555 e-1(5.27 e-2)-$ & $1.3471 e-1(3.73 e-2) \approx$ \\
UF9 & 3 & $1.3775 e-1(5.50 e-2)$ & $3.3876 e-1(1.27 e-1)-$ & $4.1963 e-1(5.45 e-2)-$ & $3.9328 e-1(1.40 e-1)-$ & $1.9027 e-1(5.71 e-2)-$ \\
UF10 & 3 & $3.3628 e-1(4.64 e-2)$ & $4.4792 e-1(6.74 e-2)-$ & $8.5001 e-1(2.25 e-1)-$ & $4.0483 e-1(7.23 e-2)-$ & $5.4458 e-1(5.32 e-2)-$ \\
& $+/-1 \approx$ & $0 / 10 / 0$ & $0 / 10 / 0$ & $0 / 10 / 0$ & $3 / 5 / 2$ \\
\hline
\end{tabular}

$'+{ }^{\prime}, \quad$ ' $-{ }^{\prime}$ and ${ }^{\prime} \approx{ }^{\prime}$ indicates that the result is significantly better, significantly worse, and statistically similar to that obtained by DEREA, respectively.

MOEAs estimate the density of each individual in the population based on the position of the individual's neighbors. Therefore, the long-term existence of low-convergence individuals will cause the disappearance of some highconvergence individuals in the limitation of population size in the environmental selection. When estimating the surrounding area density around in any individual in a population, reflecting the relative proximity of the individual to the PF by DER will shift the position of other individuals based their convergence. This will better maintain the diversity of solutions. Hence, DEREA adopts DER strategy in order to select the convergence of the individual in the population.

\section{Experimental Results and Analysis}

In this section, the suggested DEREA with some state-of-theart MOEAs was designed for solving MOPs to demonstrate the performance of the DER, and these classical MOEAs include GDE3 [47], dMOPSO [48], MOPSO [49], MOEA/D [14], MOEA/DDE [50], NSGA-II [6], PESAII [7], SMEA [51], and SPEA2 [8]. The main step of the experimental process includes

(1) Firstly, the experimental settings used in this paper are illustrated

(2) Secondly, each algorithm is run 30 times independently for each test question, and the Wilcoxon rank sum test is adopted to compare the results obtained by DEREA and those by others compared algorithms at a significance level of 0.05

In the experiments, test problems include multiple widely used test suites that are employed and DTLZ1-DTLZ7, IDTLZ1, IDTLZ2 [30], WFG1-WFG9, ZDT1-ZDT6, and UF1-UF9, where seven test problems are DTLZ1-DTLZ7 from the DTLZ test suite [49], test problems WFG1-WFG9 and UF1-UF9 taken from WFG test suite [22], and UF test suites [52], respectively. Six test problems are ZDT1-ZDT6 that are taken from ZDT [14] test suites. For IDTLZ1,
IDTLZ2, DTLZ1-DTLZ7, and MaF1-MaF7, the number of decision variables is set to $n=k+M-1$, where $M$ is the objective number, $k=5$ is used for IDTLZ1 and DTLZ1, $k=10$ is used for IDTLZ2, and DTLZ2-DTLZ6, $k=20$, is used for DTLZ7. For WFG1-WFG9, the number of decision variables is set to $n=k+l, k$ is used for $(M-1)$, and $l$ is used for 10. Pareto fronts of the above part test instance are given in Table 1. For UF test suites and ZDT test suites, UF1-UF10 and ZDT1-ZDT3 test instance in the number of decision variables is set to 30 , and ZDT4 with ZDT6 and ZDT5 corresponds to the setting $n=10$ and $n=80$, where UF test suites contain 10 continuous multiobjective optimization problems with constraints. All the compared MOEAs in this paper are implemented based on MOEA platform PlatEMO [53].

\subsection{Experimental Setting}

(1) Setting for crossover and mutation operators: The simulated polynomial mutation [40] and binary crossover (SBX) [41] are applied in all MOEAs. The probabilities of crossover and mutation are set to 1.00 and $1 / D$ (where $D$ denoting the number of decision variables). The distribution indexes of both SBX and polynomial mutation are set to 20

(2) Population size: The population sizes of the algorithm for experimental comparison are determined by the number of reference vectors or reference points in this paper. For problems with $M \geq 3$, the population size is generated by a two-layer vector generation strategy [54]. For the accuracy of the comparison results, the specific setting of the population size of the comparison algorithms is summarized in Table 2, and the population sizes are also set according to Table 2 for the proposed DEREA

(3) Performance metrics: In these experiments, the widely used hypervolume (HV) and the inverted generational distance (IGD) were adopted to as 


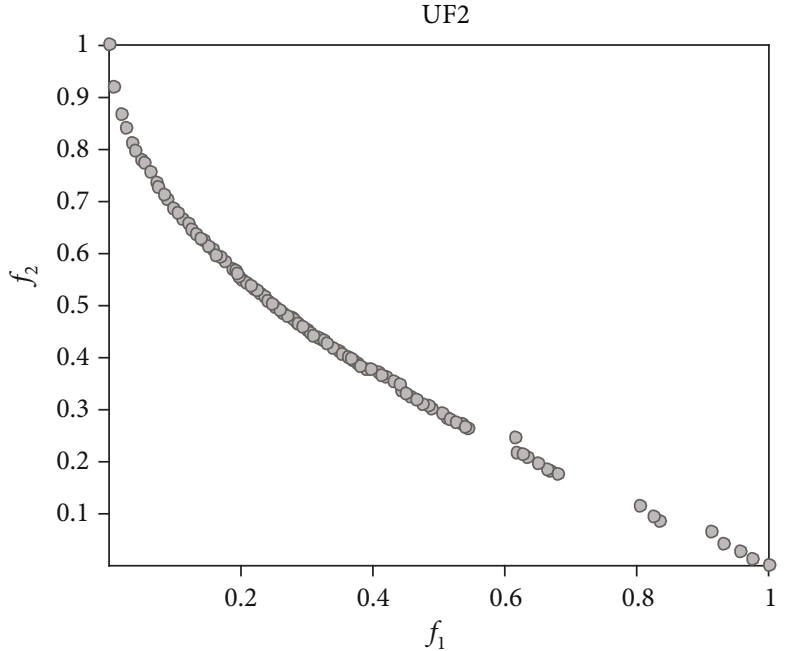

(a) DEREA

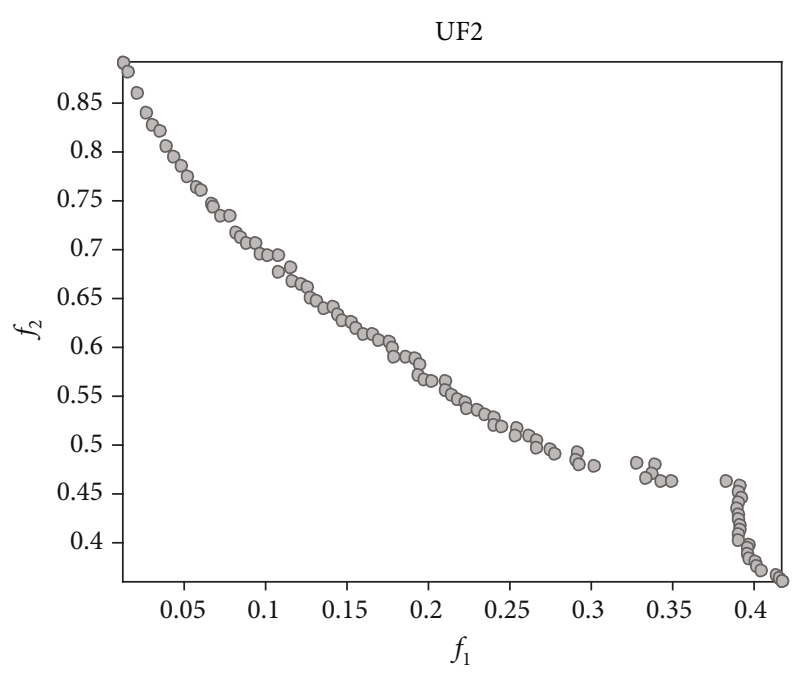

(c) MOEA/D [14]

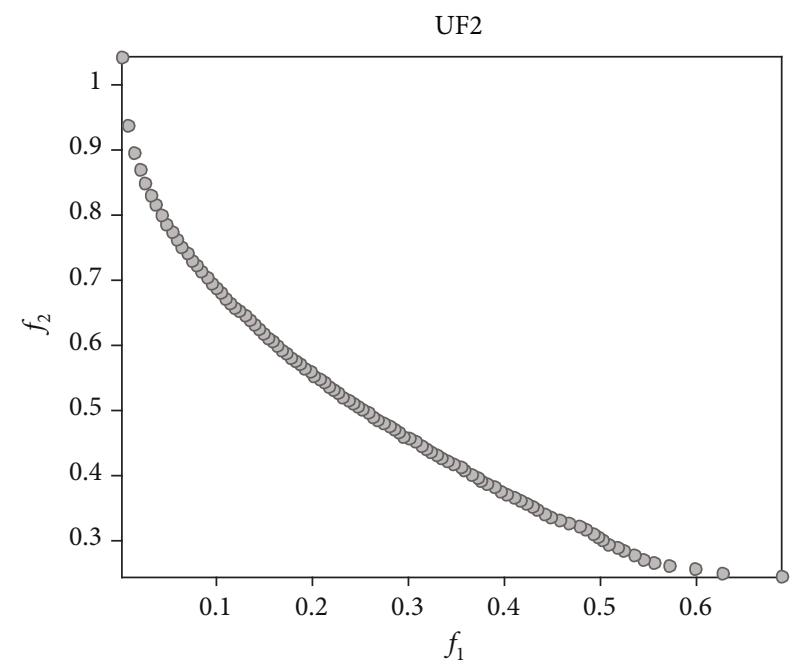

(e) MOEA/DDE [50]

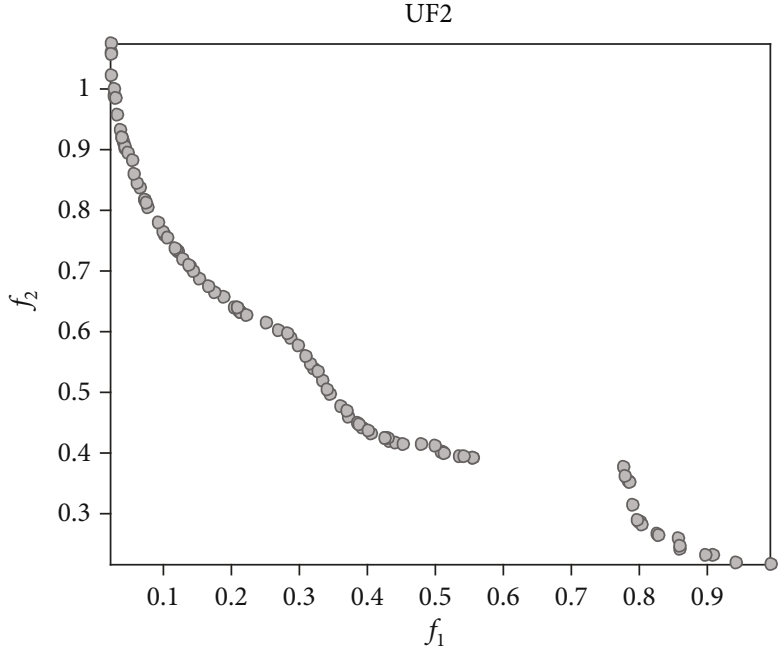

(b) MOPSO [49]

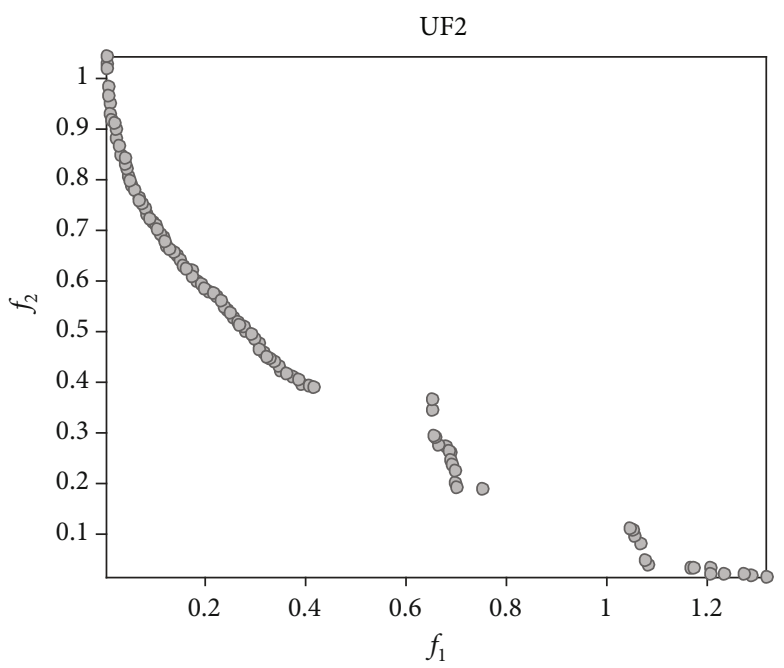

(d) GDE3 [43]

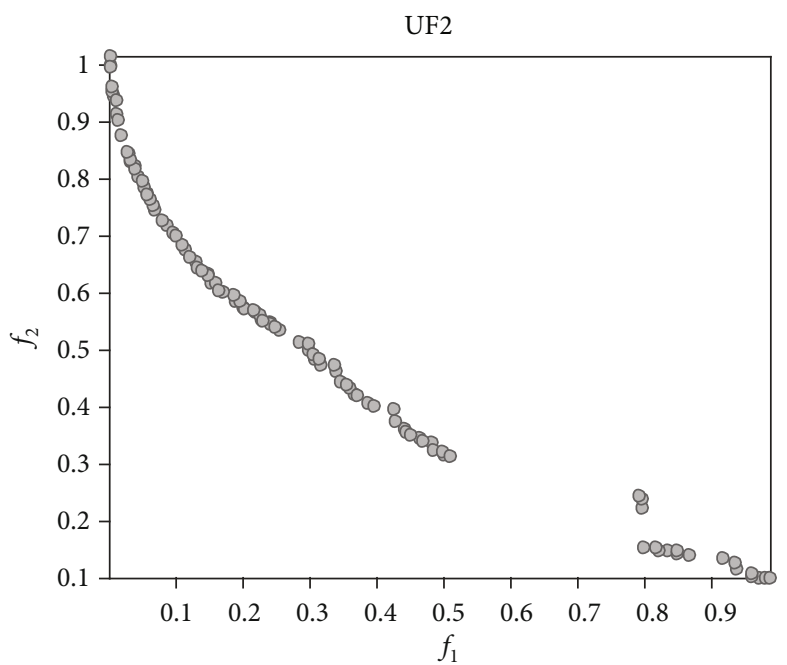

(f) NSGA-II [6]

FIgURE 7: Continued. 


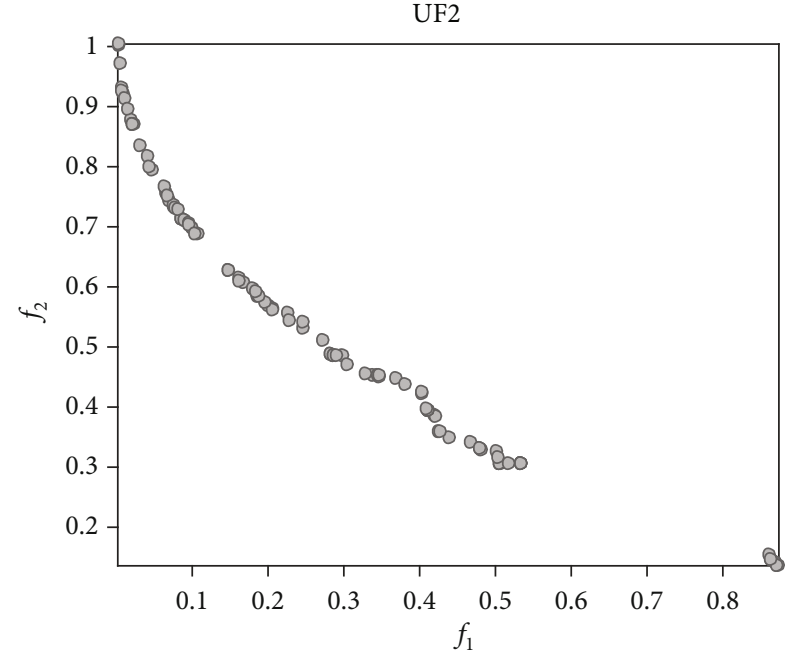

(g) PESAII [7]

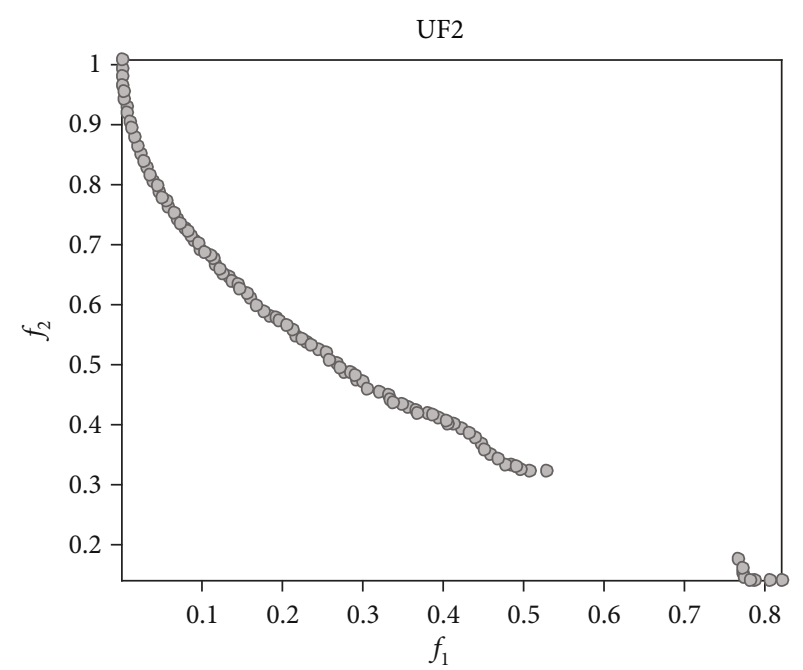

(h) SPEA2 [8]

FIGURE 7: The nondominated solutions obtained by DEREA, MOPSO, MOEA/D, GDE3, MOEA/DDE, NSGA-II, PESAII, and SPEA2 on UF2 problem with 2-objective.

TABle 5: The statistical results (mean and standard deviation) of the IGD values obtained by DEREA, PESAII, dMOPSO, NSGA-II, and MOEA/D on ZDT1-ZDT6. The best results are highlighted in blue.

\begin{tabular}{lcccccc}
\hline Problem & $M$ & DEREA & PESAII [7] & dMOPSO [48] & NSGA-II [6] & MOEA/D [14] \\
\hline ZDT1 & 2 & $4.1510 e-3(7.97 e-5)$ & $1.1847 e-2(5.24 e-3)-$ & $8.5475 e-3(2.98 e-3)-$ & $4.6078 e-3(1.47 e-4)-$ & $4.6049 e-3(6.45 e-4)-$ \\
ZDT2 & 2 & $4.6195 e-3(1.98 e-5)$ & $1.0719 e-2(1.44 e-3)-$ & $8.5282 e-2(2.06 e-1)-$ & $5.0609 e-3(2.40 e-4)-$ & $3.8946 e-3(6.24 e-5)+$ \\
ZDT3 & 2 & $2.0741 e-2(3.25 e-2)$ & $6.5135 e-2(6.04 e-2)-$ & $3.1740 e-2(9.28 e-2)-$ & $3.0418 e-2(3.52 e-2)-$ & $2.4470 e-2(2.07 e-2)-$ \\
ZDT4 & 2 & $4.8255 e-3(6.43 e-4)$ & $1.1187 e-2(1.86 e-3)-$ & $7.3167 e-3(2.21 e-3)-$ & $4.8779 e-3(5.48 e-4) \approx$ & $1.0252 e-2(2.48 e-3)-$ \\
ZDT5 & 2 & $1.1021 e+0(2.90 e-1)$ & $4.9351 e-1(2.36 e-1) \approx$ & $0.0000 e+0(0.00 e-0)-$ & $1.7390 e-2(3.69 e-2) \approx$ & $1.2512 e+1(3.96 e-1)-$ \\
ZDT6 & 2 & $3.3452 e-3(5.76 e-5)$ & $8.0858 e-3(1.29 e-3)-$ & $3.1043 e-3(2.03 e-6)+$ & $3.7711 e-3(1.43 e-4)-$ & $5.5360 e-3(8.50 e-4)-$ \\
& \multicolumn{2}{l}{$+1=$} & $0 / 5 / 1$ & $1 / 5 / 0$ & $0 / 4 / 2$ & $1 / 5 / 0$ \\
\hline
\end{tabular}

${ }^{\prime}+{ }^{\prime}, \quad{ }^{\prime}-{ }^{\prime}$ and ${ }^{\prime} \approx{ }^{\prime}$ indicates that the result is significantly better, significantly worse, and statistically similar to that obtained by DEREA, respectively.

performance metrics for comparisons among the experiments results obtain by each algorithm. All the objectives are normalized according to the ideal point and the worst point of the Pareto optimal front before $\mathrm{HV}$ calculation, then calculated with a reference point $(1.1,1.1, \ldots, 1.1)$. Besides, in the calculation of IGD metric, almost 5,000 uniformly distributed points are sampled on the PF by Das and Dennis's method for each test instance, where defined by the symbols '+,"-,' and' $\approx$ ' indicate that the performance metrics by others MOEA is significantly better, significantly worse, and statistically similar to that obtained by DEREA, respectively

(4) Termination condition: The termination criterion of each run is the maximal number of generations. For all test problems adopted in the experiment, set the maximal number of generations based on test problems or different number objective. For DTLZ1, DTLZ3, and WFG1-WFG9, the maximal number of generations is set to 1000. For DTLZ2, DTLZ4, and UF test suite, the maximal number of genera- tions is set to 500. For ZDT1-ZDT6 test suite, the maximal number of generations is set 300 . The maximal number of generations of each algorithm to deal with identical problems is the same

(5) Specific parameter settings in the compared algorithm: For PESA-II, the number of divisions in each objective is set to 10. For MOEA/D and MOEA/DDE, the size of weight vectors neighborhood $T$ is set to [ $0.1 \times N]$ (where $N$ is the population size), the maximum number of solutions substitute by each offspring $n_{r}$ is set to $[0.01 \times N]$, and the Tchebycheff method is employed as the aggregation function. In addition, for MOEA/DDE, the probability of selecting neighborhood solutions $\delta$ is set to 0.9. For dMOPSO, $T_{\alpha}$ denotes the age threshold that is set to 2. For SMEA, the number of neurons in each dimension of the latent space $D$ is determined by the number of dimensions $M$, the initial selforganizing maps learn rate $\tau_{0}$, and the size of neighborhood mate pools $H$ are set to 0.7 and 5, respectively. In the experimental comparisons, for GDE3, 


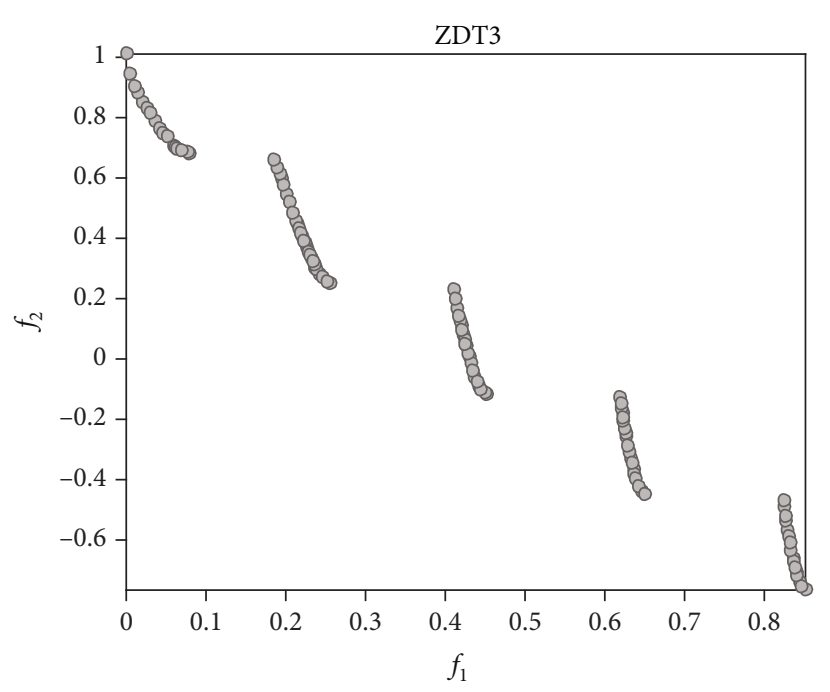

(a) DEREA

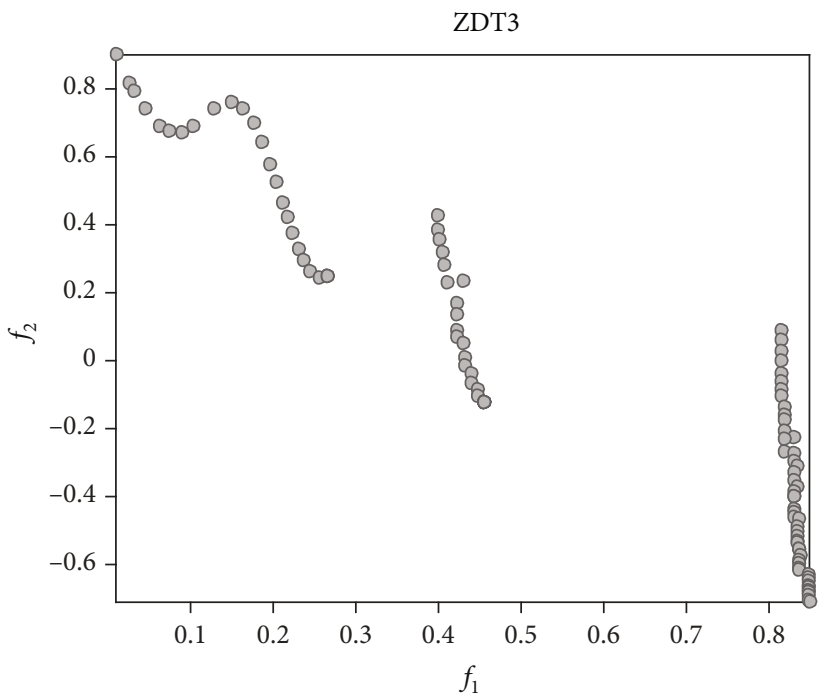

(c) MOEA/D [14]

ZDT3

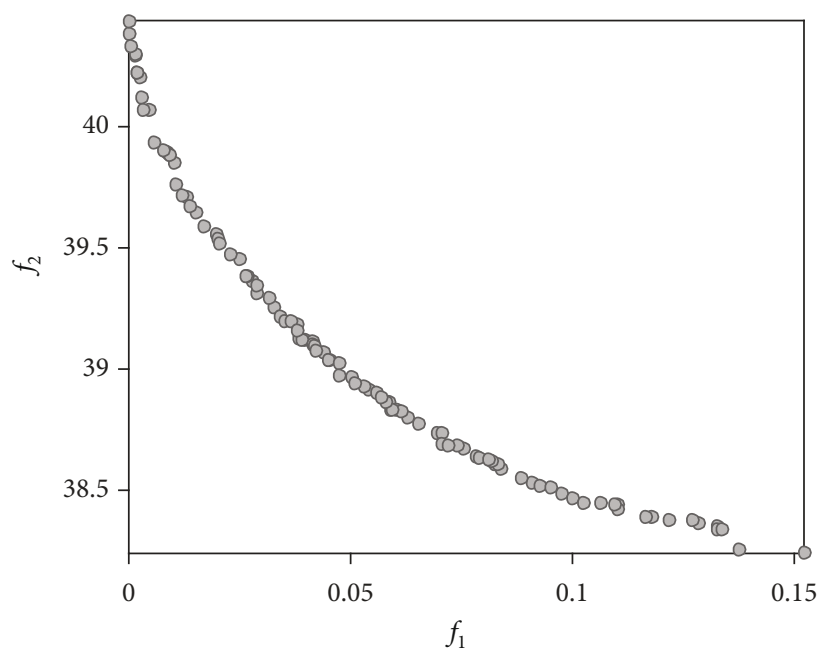

(e) MOEA/DDE [50]

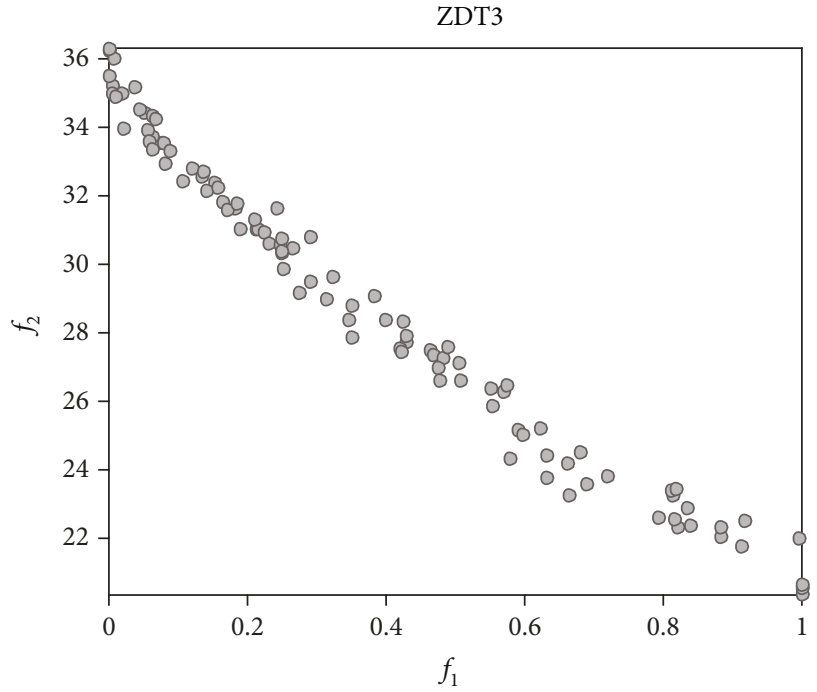

(b) MOPSO [49]

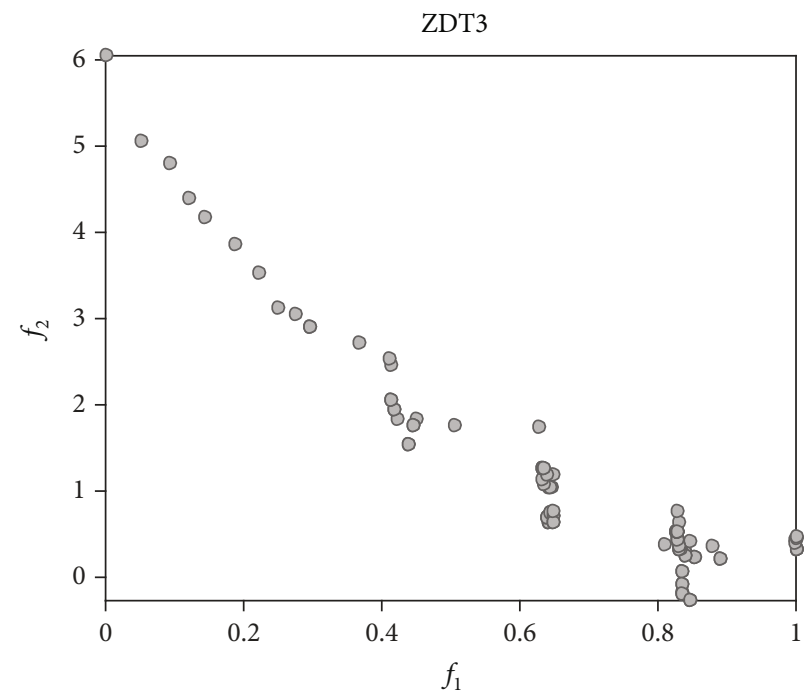

(d) GDE3 [43]

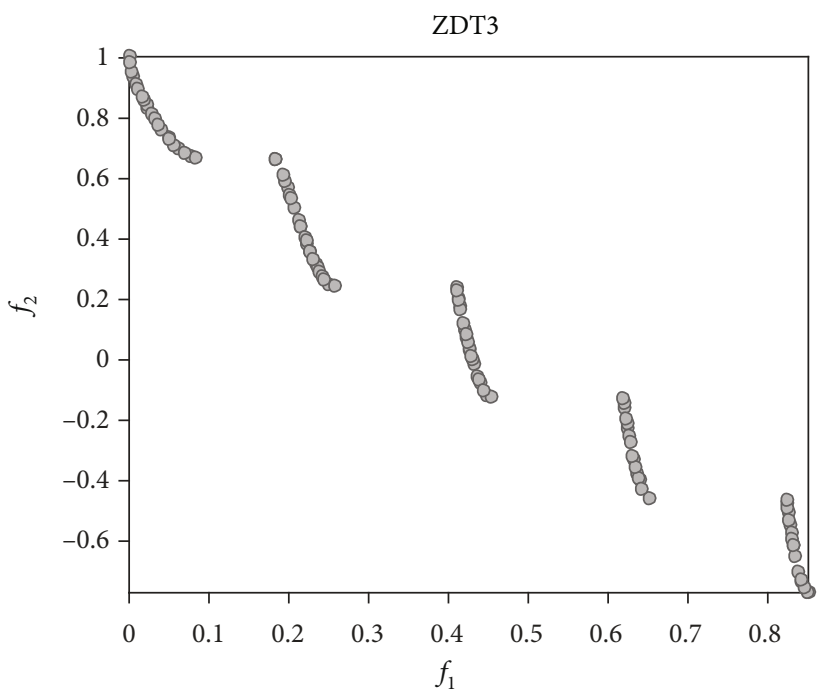

(f) NSGA-II [6]

Figure 8: Continued. 


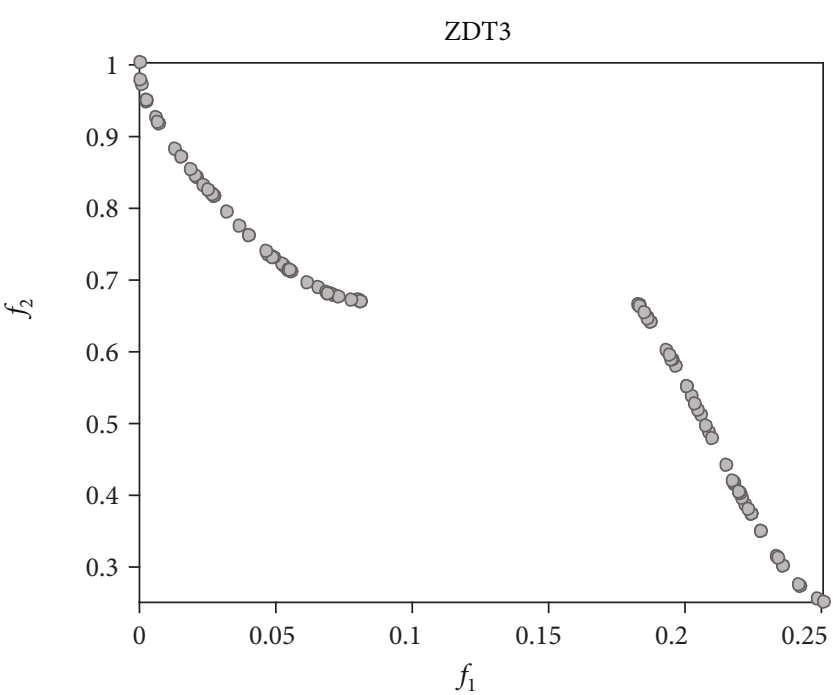

(g) PESAII [7]

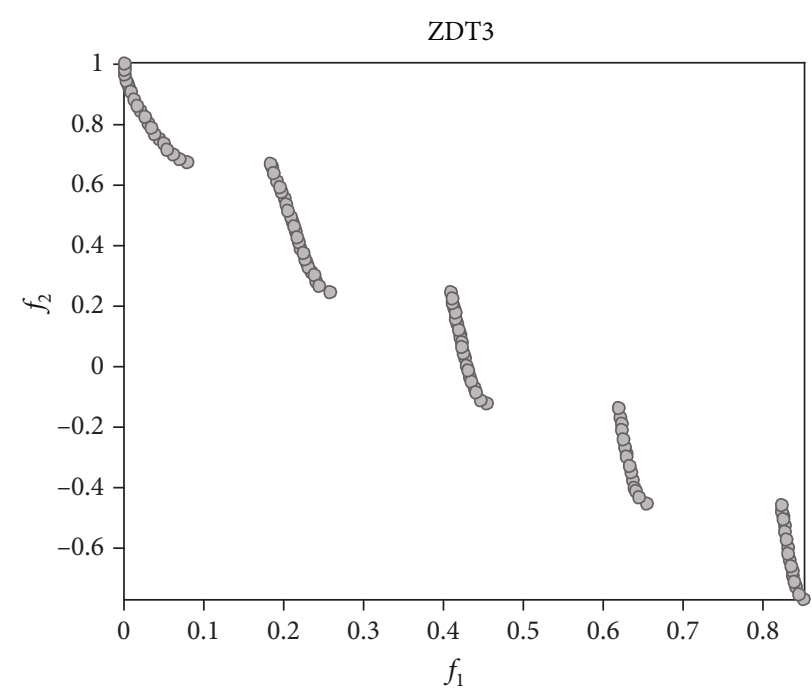

(h) SPEA2 [8]

Figure 8: The nondominated solutions obtained by DEREA, MOPSO, MOEA/D, GDE3, MOEA/DDE, NSGA-II, PESAII, and SPEA2 on ZDT3 problem with 2-objective.

NSGA-II, DEREA, and SPEA2, there are no additional specify additional parameter

\subsection{Comparisons between DEREA and Existing MOEAs for Solving MOPs}

3.2.1. Performance on WFG1-WFG9, DTLZ1-DTLZ7, and IDTLZ1-IDTLZ2. Table 3 demonstrates the HV values obtained by DEREA and other four classical MOEAs designed for solving MOPs, namely, dMOPSO, MOEA/D, NSGA-II, and SPEA2 on WFG1 to WFG9 and DTLZ1 to DTLZ7, IDTLZ1, and IDTLZ2 with two and three objectives. In general, the suggested DEREA significantly outperforms the other four MOEAs in term of the HV values. For test problems with irregular PF, Table 3 summarizes the $\mathrm{HV}$ values of each algorithm solving the test problems, and DEREA shows a performance surpass others competitors on the majority of the test instances. However, the performance of DEREA shows significance advantages with dMOPSO, MOEA/D, NSGA-II, and SPEA2 on the regular PFs. NSGA-II is outperformance by DEREA on more than 24 test instances, but it performs pretty well on WFG1 and IDTLZ1. MOEA/D and SPEA2 achieve competitive results on IDTLZ problems. The dMOPSO does not have an advantage and is surpassed by DEREA on almost all the test problems. The performance will become poor when algorithms solving with irregular PF test problems, and algorithms with density estimation (i.e., DEREA) indicate clear improvement over the others competitor MOEAs. However, the performance of MOEA/D, NSGA-II, and SPEA2 deteriorates significantly, being inferior to both DEREA on the most test instance. This phenomenon resulted from the fact that the Pareto fronts of these test instances are complicated, disconnected, or degenerate.

To visually understand the experimental results, Figure 4 plots the solution sets on the 3 -objective DTLZ1. SMEA per- forms poorly on this test problem, and the selection solutions do not converge to PF. The other several MOEAs indicate high performance in terms of converges. MOEA/D and MOEA/DDE reach the PF sparse area and obtain a distributed solution sets, but its selection solutions may not be close to the true PF, which illustrates why it has small HV value. The solutions are uniformly distributed on PF by DEREA obtained. It is easy to see that the solution obtained by dMOPSO, NSGA-II, PESAII, and SPEA2 has poor performance, which due to solutions located near the PF boundaries. Figure 5 plots the final solutions on the 3-objective WFG5. NSGA-II, PESAII, and SPEA2 converge to the PF region but its solutions fail to uniformly distribute. Due to some dominant solutions that were deleted with that algorithm selective solution only by individual position information, SMEA indicates poor performance in terms of coverage. The other three MOEAs have their own traits in the distribution of the solutions, and they obtain smaller $\mathrm{HV}$ values. dMOPSO selected optimal solutions of away from the PF and MOEA/D obtain solutions located near the PF boundaries. And MOEA/DDE has poor performance with diversity.

The final solutions on the 3-objective IDTLZ2 problem plotted in three-dimensional space are provided in Figure 6. dMOPSO, NSGA-II, PESAII, and SMEA all converge to the $\mathrm{PF}$ area, and however, fail to manager population diversity. The other three competitors indicate clear advantages in preserving diversity. These algorithms show performance that are similar to DEREA, but the best HV value by DEREA was obtained.

3.2.2. Performance on UF1-UF10. The results on problems with UF in terms of IGD are presented in Table 4. DEREA performs the best among the five algorithms. It obtains the best results on ten test instances. NSGA-II and MOEA/DDE are also competitive with DEREA on the majority of the test instances, i.e., UF1, UF2, and UF7. Other competitors (i.e., 
PESAII and dMOPSO) perform inferior to the above algorithms, where PESAII and dMOPSO indicate performance poorly on the most test instances.

To further observe the differences among solutions set obtained by the several compared algorithms, add three classical algorithms (i.e., MOEA/D, GDE3 and SPEA2). Figure 7 depicts the objective values of nondominated solution sets with the IGD value. It can be clearly seen that the solution sets obtained by DEREA and MOEA/DDE have shown good performance, and this is why IGD values are similar. The performance of other algorithms is similar, but except MOEA/D, which it selected optimal solutions that fail converge to the PF. Comparing this algorithm in UF2 instance DEREA has indicated a slight advantage.

3.2.3. Performance on ZDT1-ZDT6. Table 5 presents the mean and standard deviation of the IGD values of the final solutions by each algorithm obtained for ZDT test suites. Table 5 reveals that in terms of IGD, the final solutions by DEREA are better than the other algorithms for ZDT1, ZDT3, ZDT4, and ZDT5 instances. DEREA performance surpasses the other four algorithms.

To facilitate visual comparison, Figure 8 shows the final solutions of a run of the several algorithms. Clearly, the solutions of DEREA have good balance between diversity and convergence. MOEA/D and PESAII struggle to maintain diversity, but lead to its selected solutions concentrated in a local optimum. NSGA-II and SPEA2 selected solutions show good diversity, but convergence performs slightly worse DEREA. However, MOPSO and GDE3 indicate poor performance, and the most of the solutions have a poor convergence, thus leading to its high IGD value.

\section{Conclusion}

This paper proposes density estimation ranking strategy for multiobjective optimization, namely, DEREA. In this paper, a solution ranking method named DER is proposed. The DER is introduced to adapt different shapes of Pareto fronts. In addition, the DER is the most significant contribution of this paper, changing the position of individuals by their relative proximity to the Pareto front, DER considers the convergence and diversity of each individual in the population, and the implementation of it is very simple.

Through extensive comparison of several widely use test suites, a systematic experiment was carried out. From the experiment results, the DEREA is quite competitive with the several state-of-the-art algorithms on a majority of the test suites. Moreover, due to DER are employed, the performance of DEREA is less dependent on the Pareto front shapes and is robust in solving problems having irregular Pareto fronts.

In the future, we will have a future investigation into the density estimation mechanism in the selected strategy. We also want the future how to deal with more challenging problems, some of which are not easy to obtain during the search process. And it is also meaningful to study many objective optimization problems.

\section{Data Availability}

The dataset used to support the findings of this study are available from the corresponding author upon request.

\section{Conflicts of Interest}

The authors have declared that no competing interests exist.

\section{References}

[1] E. J. S. Pires, P. B. de Moura Oliveira, and J. A. T. Machado, "Multi-objective Genetic Manipulator Trajectory Planner," in Workshops on Applications of Evolutionary Computation, Springer, Berlin, Heidelberg, 2004.

[2] C. Wang, Z. Ye, and W. Wang, "A Multi-Objective Optimization and Hybrid Heuristic Approach for Urban Bus Route Network Design," IEEE Access, vol. 8, pp. 12154-12167, 2020.

[3] A. Asrari, S. Lotfifard, and M. S. Payam, "Pareto dominancebased multiobjective optimization method for distribution network reconfiguration," IEEE Transactions on Smart Grid, vol. 7, no. 3, pp. 1401-1410, 2016.

[4] Z. Zhao, F. Lin, X. Wang, and R. Liu, "Electricity price decision-making method of electricity selling company based on multi-objective optimization and min-max regret theory," Journal of Physics: Conference Series, vol. 1650, no. 3, article 032171, 2020.

[5] D. W. Corne and J. D. Knowles, "Techniques for highly multiobjective optimisation: some nondominated points are better than others," in Proceedings of the International Conference on Genetic and Evolutionary Computation, pp. 773-780, London, England, 2007.

[6] K. Deb, A. Pratap, S. Agarwal, and T. Meyarivan, "A fast and elitist multiobjective genetic algorithm: NSGA-II," IEEE Transactions on Evolutionary Computation, vol. 6, no. 2, pp. 182-197, 2002.

[7] D. Corne, N. Jerram, J. D. Knowles, M. Oates, and J. Martin, "PESA-II: Region-based Selection in Evolutionary Multiobjective Optimization," in Proceedings of the 3rd annual conference on genetic and evolutionary computation, pp. 283-290, San Francisco, CA, USA, 2001.

[8] E. Zitzler, M. Laumanns, and L. Thiele, "SPEA2: Improving the strength Pareto evolutionary algorithm," Tech. Rep. TIKReport 103, ETH Zurich, 2001.

[9] K. Deb, M. Mohan, and S. Mishra, "Towards a Quick Computation of Well-Spread Pareto-Optimal Solutions," in Evolutionary Multi-Criterion Optimization, Second International Conference, Emo, Faro, Portugal, April. Springer-Verlag, 2003.

[10] C. Yue, B. Qu, and L. Jing, "A Multiobjective particle swarm optimizer using ring topology for solving multimodal Multiobjective problems," IEEE Transactions on Evolutionary Computation, vol. 22, no. 5, pp. 805-817, 2018.

[11] T. Cheng, M. Chen, P. J. Fleming, Z. Yang, and S. Gan, "A novel hybrid teaching learning based multi-objective particle swarm optimization," Neurocomputing, vol. 222, pp. 11-25, 2016.

[12] H. L. Liu, F. Gu, and Q. Zhang, "Decomposition of a multiobjective optimization problem into a number of simple multiobjective sub-problems," IEEE Transactions on Evolutionary Computation, vol. 18, no. 3, pp. 450-455, 2014.

[13] T. Murata, "Specification of genetic search directions in cellular multi-objective genetic algorithms," in International 
Conference on Evolutionary Multi-Criterion Optimization, pp. 82-95, Berlin, Heidelberg, 2001.

[14] Q. Zhang and H. Li, "MOEA/D: a multiobjective evolutionary algorithm based on decomposition," IEEE Transactions on Evolutionary Computation, vol. 11, no. 6, pp. 712-731, 2008.

[15] R. Cheng, Y. Jin, M. Olhofer, and B. Sendhoff, "A reference vector guided evolutionary algorithm for many-objective optimization," IEEE Transactions on Evolutionary Computation, vol. 20, no. 5, pp. 773-791, 2016.

[16] X. He, Y. Zhou, Z. Chen, and Q. Zhang, "Evolutionary manyobjective optimization based on dynamical decomposition," IEEE Transactions on Evolutionary Computation, vol. 23, no. 3, pp. 361-375, 2019.

[17] K. Deb and H. Jain, "An evolutionary many-objective optimization algorithm using reference-point-based nondominated sorting approach, part I: solving problems with box constraints," IEEE Transactions on Evolutionary Computation, vol. 18, no. 4, pp. 577-601, 2014.

[18] R. Cheng, Y. Jin, K. Narukawa, and B. Sendhoff, "A multiobjective evolutionary algorithm using Gaussian process-based inverse modeling," IEEE Transactions on Evolutionary Computation, vol. 19, no. 6, pp. 838-856, 2015.

[19] G. Li, W. Wang, W. Zhang, Z. Wang, H. Tu, and W. You, "Grid search based multi-population particle swarm optimization algorithm for multimodal multi-objective optimization," Swarm and Evolutionary Computation, vol. 62, article id 100843, 2021.

[20] M. Wu, K. Li, S. Kwong, Q. Zhang, and J. Zhang, "Learning to decompose: a paradigm for decomposition-based multiobjective optimization," IEEE Transactions on Evolutionary Computation, vol. 23, no. 3, pp. 376-390, 2019.

[21] H. L. Liu, F. Gu, and Q. Zhang, "Decomposition of a multiobjective optimization problem into a number of simple multiobjective subproblems," IEEE Transactions on Evolutionary Computation, vol. 18, no. 3, pp. 450-455, 2014.

[22] S. Jiang and S. Yang, "A strength Pareto evolutionary algorithm based on reference direction for multi-objective and many-objective optimization," IEEE Transactions on Evolutionary Computation, vol. 21, no. 3, pp. 329-346, 2017.

[23] N. Beume, B. Naujoks, and M. Emmerich, "SMS-EMOA: multiobjective selection based on dominated hypervolume," European Journal of Operational Research, vol. 181, no. 3, pp. 16531669, 2007.

[24] A. Menchaca-Mendez and C. Coello, "GDE-MOEA: A new MOEA based on the generational distance indicator and $\varepsilon$ dominance," in 2015 IEEE Congress on Evolutionary Computation (CEC), pp. 947-955, Sendai, Japan, 2015.

[25] A. F. Ionescu, "Multi-objective Evolutionary Algorithms: Decomposition Versus Indicator-Based Approach," in vol. 404 of Studies inFuzziness and Soft Computing, , Springer, Cham, 2021.

[26] K. Li, S. Kwong, J. Cao, M. Li, J. Zheng, and R. Shen, “Achieving balance between proximity and diversity in multi-objective evolutionary algorithm," Information Sciences, vol. 182, no. 1, pp. 220-242, 2012.

[27] Y. Sun, G. G. Yen, and Z. Yi, "IGD Indicator-based evolutionary algorithm for many-objective optimization problems," IEEE Transactions on Evolutionary Computation, vol. 23, no. 2, pp. 173-187, 2019.

[28] R. Hernandez Gomez and C. A. Coello, "Improved metaheuristic based on the R2 indicator for many-objective optimiza- tion," in Proceedings of the 2015 International Conference on Genetic and Evolutionary Computation, pp. 679-686, New York, NY, USA, 2015.

[29] J. Bader and E. Zitzler, "HypE: An algorithm for fast Hypervolume-based many-objective optimization," Evolutionary Computation, vol. 19, no. 1, pp. 45-76, 2011.

[30] Y. Tian, R. Cheng, X. Zhang, F. Cheng, and Y. Jin, “An Indicator Based Multi-Objective Evolutionary Algorithm with Reference Point Adaptation for Better Versatility," IEEE Transactions on Evolutionary Computation, vol. 22, pp. 609622, 2017.

[31] X. Ma, F. Liu, Y. Qi et al., "A multiobjective evolutionary algorithm based on decision variable analyses for multiobjective optimization problems with large-scale variables," IEEE Transactions on Evolutionary Computation, vol. 20, no. 2, pp. 275-298, 2016.

[32] H. Li, Q. Zhang, and J. Deng, "Biased multiobjective optimization and decomposition algorithm," IEEE Transactions on Cybernetics, vol. 47, no. 1, pp. 52-66, 2017.

[33] G. Chen and J. Li, "A diversity ranking based evolutionary algorithm for multi-objective and many-objective optimization," Swarm and Evolutionary Computation, vol. 48, pp. 274-287, 2019.

[34] Y. Liang, H. Huang, Z. Cai, and Z. Hao, "Multiobjective evolutionary optimization based on fuzzy multicriteria evaluation and decomposition for image matting," IEEE Transactions on Fuzzy Systems, vol. 27, no. 5, pp. 1100-1111, 2019.

[35] H. Wang, L. Jiao, and X. Yao, "Two_Arch2: An improved Two-archive algorithm for many-objective optimization," IEEE Transactions on Evolutionary Computation, vol. 19, no. 4 , pp. 524-541, 2015.

[36] Y. Liu, D. Gong, J. Sun, and Y. Jin, “A many-objective evolutionary algorithm using a one-by-one selection strategy," IEEE Transactions on Cybernetics, vol. 47, no. 9, pp. 2689-2702, 2017.

[37] M. Li, S. Yang, and X. Liu, "Pareto or non-Pareto: bi-criterion evolution in multiobjective optimization," IEEE Transactions on Evolutionary Computation, vol. 20, no. 5, pp. 645-665, 2016.

[38] H. Ishibuchi, Y. Setoguchi, H. Masuda, and Y. Nojima, "Performance of decomposition-based many-objective algorithms strongly depends on Pareto front shapes," IEEE Transactions on Evolutionary Computation, vol. 21, no. 2, pp. 169-190, 2017.

[39] C. M. Anderson-Cook, "Practical Genetic Algorithms," Journal of the American Statistical Association, vol. 100, no. 471, pp. 1099-1099, 2005.

[40] K. Deb and M. Goyal, "A combined genetic adaptive search (gene AS) for engineering design," Computer Science and Informatics, vol. 26, no. 4, pp. 30-45, 1996.

[41] K. Deb and R. B. Agrawal, "Simulated binary crossover for continuous search space," Complex Systems, vol. 9, no. 4, pp. 115-148, 1995.

[42] C. A. Coello Coello and E. Mezura Montes, "Constraint-handling in genetic algorithms through the use of dominancebased tournament selection," Advanced Engineering Informatics, vol. 16, no. 3, pp. 193-203, 2002.

[43] G. G. Yen and Haiming Lu, "Dynamic multiobjective evolutionary algorithm: adaptive cell-based rank and density estimation," IEEE Transactions on Evolutionary Computation, vol. 7, no. 3, pp. 253-274, 2003. 
[44] M. Li, J. Zheng, K. Li, Q. Yuan, and R. Shen, "Enhancing diversity for average ranking method in evolutionary manyobjective optimization," in Parallel Problem Solving from Nature, PPSN XI, pp. 647-656, Springer, 2010.

[45] M. Li, J. Zheng, R. Shen, K. Li, and Q. Yuan, "A grid-based fitness strategy for evolutionary many-objective optimization," in Proceedings of the 12th annual conference on Genetic and evolutionary computation - GECCO '10, pp. 463-470, New York, NY, USA, 2010.

[46] M. Li, S. Yang, and X. Liu, "Shift-based density estimation for Pareto-based algorithms in many-objective optimization," IEEE Transactions on Evolutionary Computation, vol. 18, no. 3, pp. 348-365, 2014.

[47] S. Kukkonen and J. Lampinen, "GDE3: The third Evolution Step of Generalized Differential Evolution," in 2005 IEEE Congress on Evolutionary Computation, vol. 1, pp. 443-450, Edinburgh, UK, September 2005.

[48] S. Z. Martinez and C. A. C. Coello, "A multi-objective particle swarm optimizer based on decomposition," in Proceedings of the 13th Annual Conference on Genetic and Evolutionary Computation, pp. 69-76, New York, NY, USA, 2011.

[49] C. A. Coello and M. S. Lechuga, "MOPSO: a proposal for multiple objective particle swarm optimization," in Proceedings of the 2002 Congress on Evolutionary Computation. CEC'02 (Cat. No.02TH8600), pp. 1051-1056, Honolulu, HI, USA, 2002.

[50] H. Li and Q. Zhang, "Multiobjective optimization problems with complicated Pareto sets, MOEA/D and NSGA-II," IEEE Transactions on Evolutionary Computation, vol. 13, no. 2, pp. 284-302, 2009.

[51] H. Zhang, A. Zhou, S. Song, Q. Zhang, X. Z. Gao, and J. Zhang, "A self-organizing multiobjective evolutionary algorithm," IEEE Transactions on Evolutionary Computation, vol. 20, no. 5, pp. 792-806, 2016.

[52] Q. F. Zhang, W. D. Liu, and H. Li, "The performance of a new version of MOEA/D on CEC09 unconstrained MOP test instances," in IEEE Congress on Evolutionary Computation, Trondheim, Norway, 2009.

[53] Y. Tian, R. Cheng, X. Zhang, and Y. Jin, "PlatEMO: a MATLAB platform for evolutionary multi-objective optimization [educational forum]," IEEE Computational Intelligence Magazine, vol. 12, no. 4, pp. 73-87, 2017.

[54] K. Li, Q. Zhang, and S. Kwong, "An evolutionary manyobjective optimization algorithm based on dominance and decomposition," IEEE Transactions on Evolutionary Computation, vol. 19, no. 5, pp. 694-716, 2015. 